\title{
Statistical State Dynamics of Weak Jets in Barotropic Beta-Plane Turbulence
}

\author{
NiKOLAOS A. BAKAS \\ Laboratory of Meteorology and Climatology, Department of Physics, University of Ioannina, Ioannina, Greece \\ NAVID C. CONSTANTINOU \\ Scripps Institution of Oceanography, University of California, San Diego, La Jolla, California, and Research \\ School of Earth Sciences, and Australian Research Council Centre of Excellence for Climate Extremes, \\ Australian National University, Canberra, Australian Capital Territory, Australia \\ Petros J. IOANNOU \\ Department of Physics, National and Kapodistrian University of Athens, Athens, Greece
}

(Manuscript received 26 May 2018, in final form 23 October 2018)

\begin{abstract}
Zonal jets in a barotropic setup emerge out of homogeneous turbulence through a flow-forming instability of the homogeneous turbulent state (zonostrophic instability), which occurs as the turbulence intensity increases. This has been demonstrated using the statistical state dynamics (SSD) framework with a closure at second order. Furthermore, it was shown that for small supercriticality the flow-forming instability follows Ginzburg-Landau (G-L) dynamics. Here, the SSD framework is used to study the equilibration of this flowforming instability for small supercriticality. First, we compare the predictions of the weakly nonlinear G-L dynamics to the fully nonlinear SSD dynamics closed at second order for a wide range of parameters. A new branch of jet equilibria is revealed that is not contiguously connected with the $\mathrm{G}-\mathrm{L}$ branch. This new branch at weak supercriticalities involves jets with larger amplitude compared to the ones of the G-L branch. Furthermore, this new branch continues even for subcritical values with respect to the linear flow-forming instability. Thus, a new nonlinear flow-forming instability out of homogeneous turbulence is revealed. Second, we investigate how both the linear flow-forming instability and the novel nonlinear flow-forming instability are equilibrated. We identify the physical processes underlying the jet equilibration as well as the types of eddies that contribute in each process. Third, we propose a modification of the diffusion coefficient of the G-L dynamics that is able to capture the evolution of weak jets at scales other than the marginal scale (side-band instabilities) for the linear flow-forming instability.
\end{abstract}

\section{Introduction}

Robust eddy-driven zonal jets are ubiquitous in planetary atmospheres (Ingersoll 1990; Ingersoll et al. 2004; Vasavada and Showman 2005). Laboratory experiments, theoretical studies, and numerical simulations show that small-scale turbulence self-organizes into large-scale coherent structures, which are predominantly zonal and, furthermore, that the small-scale turbulence supports the jets against eddy mixing (Starr 1968; Huang and Robinson 1998; Read et al. 2007; Salyk et al. 2006). One of the simplest models, which is a test

Corresponding author: Navid Constantinou, navid.constantinou@ anu.edu.au bed for theories regarding turbulence self-organization, is forced-dissipative barotropic turbulence on a beta plane.

An advantageous framework for understanding coherent zonal jet self-organization is the study of the statistical state dynamics (SSD) of the flow. SSD refers to the dynamics that governs the statistics of the flow rather than the dynamics of individual flow realizations. However, evolving the hierarchy of the flow statistics of a nonlinear dynamics soon becomes intractable; a turbulence closure is needed. Unlike the usual paradigm of homogeneous isotropic turbulence, when strong coherent flows coexist with the incoherent turbulent field, the SSD of the turbulent flow is well captured by a second-order closure (Farrell and Ioannou 2003, 2007, 2009; Tobias et al. 2011; Srinivasan and Young 2012; 
Bakas and Ioannou 2013a; Tobias and Marston 2013; Constantinou et al. 2014a,b; Thomas et al. 2014; AitChaalal et al. 2016; Constantinou et al. 2016; Farrell et al. 2016; Farrell and Ioannou 2017; Fitzgerald and Farrell 2018a, 2019; Frishman and Herbert 2018; Bakas and Ioannou 2019b). Such a second-order closure comes in the literature under two names: stochastic structural stability theory (S3T; Farrell and Ioannou 2003) and cumulant expansion at second order (CE2; Marston et al.2008). We refer to this second-order closure as S3T.

Using the S3T second-order closure it was first theoretically predicted that zonal jets in barotropic beta-plane turbulence emerge spontaneously out of a background of homogeneous turbulence through an instability of the SSD (Farrell and Ioannou 2007; Srinivasan and Young 2012). That is, S3T predicts that jet formation is a bifurcation phenomenon, similar to phase transitions, that appears as the turbulence intensity crosses a critical threshold. This prediction comes in contrast with the usual theories for zonal jet formation that involve anisotropic arrest of the inverse energy cascade at the Rhines scale (Rhines 1975; Vallis and Maltrud 1993). Jet emergence as a bifurcation was subsequently confirmed by comparison of the analytic predictions of the S3T closure with direct numerical simulations (Constantinou et al. 2014a; Bakas and Ioannou 2014). This flowforming SSD instability is markedly different from hydrodynamic instability in which the perturbations grow in a fixed mean flow. In the flow-forming instability, both the coherent mean flow and the incoherent eddy field are allowed to change. The instability manifests as follows: a weak zonal flow that is inserted in an otherwise homogeneous turbulent field organizes the incoherent fluctuations to coherently reinforce the zonal flow. This instability has analytic expression only in the SSD, and we therefore refer to this new kind of instabilities as "SSD instabilities." In particular, the flow-forming SSD instability of the homogeneous turbulent state to zonal jet mean-flow perturbations is also referred to as "zonostrophic instability" (Srinivasan and Young 2012).

Kraichnan (1976) suggested that the large-scale mean flow is supported by small-scale eddies. Indeed, when the large scales dominate the eddy field (i.e., when the large-scale shear time $\tau_{m}$ is far shorter than the eddy turnover time $\tau_{e}$ ), the small-scale eddies have the tendency to flux momentum and support large-scale mean flows (Shepherd 1987; Huang and Robinson 1998; Chen et al. 2006; Holloway 2010; Frishman and Herbert 2018). Under such circumstances, we expect the S3T secondorder closure of the SSD to be accurate. Furthermore, Bouchet et al. (2013) showed that in the limit $\tau_{e} / \tau_{m} \rightarrow \infty$ the SSD of large-scale jets in equilibrium with their eddy field are governed exactly by a second-order closure.

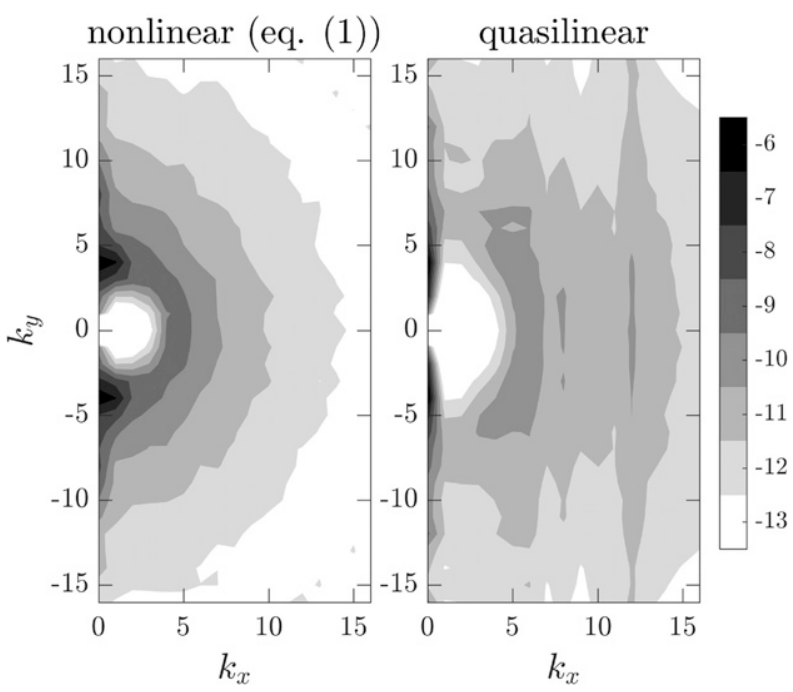

FIG. 1. Second-order closure captures the mean-flow dynamics despite differences in structure of eddy spectra. Shown here are the energy spectra for (left) a fully nonlinear simulation [see (1)] and (right) its quasilinear approximation (i.e., employing the secondorder closure). Both simulations form four jets of similar strength. Setup as described in section 5 with $\beta /\left(k_{f} r\right)=70$ and $\varepsilon /\left(k_{f}^{2} r^{3}\right)=$ $4 \times 10^{5}$. Contours are in logarithmic scale and are the same for both panels.

Recent studies revealed that the second-order closure remains accurate even at moderate scale separation between $\tau_{m}$ and $\tau_{e}$ (e.g., Srinivasan and Young 2012; Marston et al. 2019, 2016; Frishman et al. 2017; Frishman and Herbert 2018). That is, the second-order closure manages to reproduce fairly accurately the structure of the mean flow even though there could be differences in the eddy spectra and the concomitant eddy correlations; see, for example, Fig. 1.

However, surprisingly, S3T remains accurate even at a perturbative level, that is, when the mean flows/jets are just emerging with $\tau_{e} / \tau_{m} \rightarrow 0$ [the exact opposite limit of Bouchet et al. (2013)]. This perturbative-level agreement is reported by Constantinou et al. (2014a) and Bakas and Ioannou (2013a, 2014) for barotropic flows, by Bakas and Ioannou (2019b) for baroclinic flows, by Fitzgerald and Farrell (2018a) for vertically sheared stratified flows, by Constantinou and Parker (2018) for magnetized flows in astrophysical settings, and by Farrell et al. (2017) for the formation of spanwise varying mean flows and mean vortices (streaks-rolls) in $3 \mathrm{D}$ channel flows. The reason that the S3T second-order closure works well even for very weak mean flows should be attributed to the existence of the collective flowforming instability, which seems to overpower the disruptive eddy-eddy nonlinear interactions.

The dynamics that underlie the flow-forming SSD instability of the homogeneous state is well understood; 
Bakas and Ioannou (2013b) and Bakas et al. (2015) studied in detail this eddy-mean flow dynamics for barotropic flows and Fitzgerald and Farrell (2018b) for stratified flows. In these studies, the structures of the eddy field that produce upgradient momentum fluxes, and thus drive the instability, were determined in the appropriate limit $\tau_{\text {diss }} / \tau_{m} \rightarrow 0$, with $\tau_{\text {diss }}$ the dissipation time scale.

While the processes by which the flow-forming instability manifests are well understood, we lack comprehensive understanding of how this instability is equilibrated. For example, as the zonal jets grow, they often merge or branch to larger or smaller scales (Danilov and Gurarie 2004; Manfroi and Young 1999), multiple turbulence-jet equilibria exist (Farrell and Ioannou 2007; Parker and Krommes 2013; Constantinou et al. 2014a), and, also, transitions from various turbulent jet attractors may occur (Bouchet et al. 2019). Some outstanding questions include the following:

(i) How is the equilibration of the flow-forming instability achieved and at which amplitude for the given parameters?

(ii) What are the eddy-mean flow dynamics involved in the equilibration process, and which eddies support the finite-amplitude jets?

(iii) What type of instabilities are involved in the observed jet variability phenomenology (jet merging and branching, multiple jet equilibria, transitions between various jet attractors), and what are the eddy-mean flow dynamics involved?

To tackle these questions, Parker and Krommes (2013) first pointed out the analogy of jet formation and pattern formation (Hoyle 2006; Cross and Greenside 2009). Exploiting this analogy Parker and Krommes (2014) were able to borrow tools and methods from pattern formation theory to elucidate the equilibration process. In particular, they demonstrated that, at small supercriticality, that is, when the turbulence intensity is just above the critical threshold for jet formation, the nonlinear evolution of the zonal jets follows GinzburgLandau (G-L) dynamics. In addition, Parker and Krommes (2014) examined the quantitative accuracy of the G-L approximation by comparison with turbulent jet equilibria obtained from the fully nonlinear S3T dynamics. Having established the validity of S3T dynamics even in the limit of very weak mean flows (as we have discussed above), it is natural to then proceed studying the G-L dynamics of this flow-forming instability and its associated equilibration process. The perturbative-level agreement of the S3T predictions with direct numerical simulations of the full nonlinear dynamics argues that the study of the equilibration of the flow-forming instability using the G-L dynamics is well founded.
In this work, we revisit the small-supercriticality regime of Parker and Krommes (2014). We thoroughly test the validity of the G-L approximation through a comparison with the fully nonlinear SSD closed at second order for a wide range of parameter values (section 5). Apart from the equilibrated flow-forming instability of the homogeneous turbulent state, which is governed by the G-L dynamics, we discover that an additional branch of jet equilibria exists for large values of $\beta /\left(k_{f} r\right)$ ( $\beta$ is the planetary vorticity gradient, $r=1 / \tau_{\text {diss }}$ is the linear dissipation rate, and $1 / k_{f}$ is the length scale of the forcing). This new branch of equilibria reveals that jets emerge as a cusp bifurcation, which implies that for large $\beta /\left(k_{f} r\right)$ the emergent jets may result from a nonlinear instability (see Fig. 6a).

We investigate here the eddy-mean flow dynamics involved in the equilibration of the flow-forming instabilities, as well as those involved in the secondary side-band jet instabilities that occur (section 6). To do this, we derive the G-L equation in a physically intuitive way that allows for the comprehensive understanding of the nonlinear Landau term underlying the jet equilibration (section 4). Using methods similar to the ones developed by Bakas and Ioannou (2013b) and Bakas et al. (2015) we study the contribution of the forced eddies and their interactions in supporting the equilibrated finite-amplitude jets (section 6). Finally, to elucidate the equilibration of the new branch of jet equilibria that are not governed by the G-L dynamics, we develop an alternative reduced dynamical system that generalizes the G-L equation (section 6b). Using this reduced system we study the physical processes responsible for the equilibration of the new branch of jet equilibria.

\section{Statistical state dynamics of barotropic $\beta$-plane turbulence in the S3T second-order closure}

Consider a nondivergent flow $\mathbf{u}_{*}=\left(u_{*}, v_{*}\right)$ on a $\beta$ plane with coordinates $\mathbf{x}_{*}=\left(x_{*}, y_{*}\right) ; x_{*}$ is the zonal direction and $y_{*}$ the meridional direction. Subscript asterisks here denote dimensional variables. The flow is in an unbounded domain unless otherwise indicated. The flow is derived from a streamfunction $\psi_{*}$ via $\left(u_{*}, v_{*}\right)=\left(-\partial_{y_{*}} \psi_{*}, \partial_{x_{*}} \psi_{*}\right)$. The relative vorticity of the flow is $\zeta_{*} \stackrel{\text { def }}{=} \partial_{x *} v_{*}-\partial_{y_{*}} u_{*}=$ $\Delta_{*} \psi_{*}$, with $\Delta_{*} \stackrel{\text { def }}{=} \partial_{x_{*}}^{2}+\partial_{y_{*}}^{2}$ the Laplacian. With stochastic excitation and linear dissipation the relative vorticity evolves according to

$$
\left(\partial_{t *}+\mathbf{u}_{*} \cdot \nabla_{*}\right)\left(\zeta_{*}+\beta_{*} y_{*}\right)=-r_{*} \zeta_{*}+\sqrt{\varepsilon_{*}} \xi_{*} .
$$

Linear dissipation at the rate $r_{*}$ parameterizes Ekman drag at the surface of the planet. Turbulence is supported by the random stirring $\xi_{*}\left(\mathbf{x}_{*}, t_{*}\right)$ that injects energy in 
the flow at rate $\varepsilon_{*}$. This random stirring models vorticity sources such as convection and/or baroclinic growth processes that are absent in barotropic dynamics. The random process $\xi_{*}$ is assumed (i) to have zero mean, (ii) to be spatially and temporally statistically homogeneous, and (iii) to be temporally delta correlated but spatially correlated. Thus it satisfies

$$
\begin{aligned}
\left\langle\xi_{*}\left(\mathbf{x}_{*}, t_{*}\right)\right\rangle & =0 \quad \text { and } \\
\left\langle\xi_{*}\left(\mathbf{x}_{a^{*}}, t_{*_{1}}\right) \xi\left(\mathbf{x}_{b^{*}}, t_{*_{2}}\right)\right\rangle & =Q_{*}\left(\mathbf{x}_{a^{*}}-\mathbf{x}_{b^{*}}\right) \delta\left(t_{1 *}-t_{2^{*}}\right),
\end{aligned}
$$

with $Q_{*}$ the homogeneous spatial covariance of the forcing. Angle brackets denote ensemble averaging over realizations of the forcing. The forcing covariance is constructed by specifying a nonnegative spectral power function $\hat{Q}_{*}\left(\mathbf{k}_{*}\right)$ as

$$
Q_{*}\left(\mathbf{x}_{a^{*}}-\mathbf{x}_{b^{*}}\right)=\int \frac{d^{2} \mathbf{k}_{*}}{(2 \pi)^{2}} \hat{Q}_{*}\left(\mathbf{k}_{*}\right) e^{i \mathbf{k}_{*} \cdot\left(\mathbf{x}_{a^{*}}-\mathbf{x}_{b^{*}}\right)} .
$$

In this work, we consider isotropic forcing with spectrum

$$
\hat{Q}_{*}\left(\mathbf{k}_{*}\right)=4 \pi k_{f *} \delta\left(k_{*}-k_{f *}\right),
$$

where $k_{*} \stackrel{\text { def }}{=}\left|\mathbf{k}_{*}\right|$. The forcing in (4) excites equally all waves with total wavenumber $k_{f}$; we refer to this forcing as isotropic "ring" forcing. The forcing spectrum is normalized so that the total energy injection is $\varepsilon_{*}$.

Equation (1) is nondimensionalized using the forcing length-scale $k_{f^{*}}^{-1}$ and the dissipation time-scale $r_{*}^{-1}$. The nondimensional variables are $\zeta=\zeta_{*} / r_{*}, \mathbf{u}=\mathbf{u}_{*} /\left(k_{f *}^{-1} r_{*}\right)$, $\xi=\xi_{*} /\left(k_{f *} \sqrt{r_{*}}\right), \quad \varepsilon=\varepsilon_{*} /\left(k_{f *}^{-2} r_{*}^{3}\right), \quad \beta=\beta_{*} /\left(k_{f *} r_{*}\right), \quad$ and $r=1$. Thus, the nondimensional version of (1) lacks all asterisks and has $r=1$. The nondimensional form of $\hat{Q}_{*}$ in (4) is obtained by dropping the asterisks and replacing $k_{f *} \mapsto 1$.

The SSD of zonal jet formation in the S3T secondorder closure comprise the dynamics of the first cumulant of the vorticity field $\bar{\zeta}(\mathbf{x}, t)$ and of the second-cumulant $C\left(\mathbf{x}_{a}, \mathbf{x}_{b}, t\right) \stackrel{\text { deff }}{=} \overline{\zeta^{\prime}\left(\mathbf{x}_{a}, t\right) \zeta^{\prime}\left(\mathbf{x}_{b}, t\right)}$.

The overbars here denote zonal average, while dashes denote fluctuations about the mean. Thus, $\bar{\zeta}=-\partial_{y} \bar{u}$, and the first cumulant of the flow can be equivalently described with $\bar{u}$. Also, the eddy covariance $C$ is therefore homogeneous in $x$ : $C\left(x_{a}-x_{b}, y_{a}, y_{b}, t\right)$. Furthermore, the zonal average is assumed to satisfy the ergodic property, that is, that the average of any quantity is equal to an ensemble average over realizations of $\xi: \overline{(\cdot)}=\langle(\cdot)\rangle$.

\footnotetext{
${ }^{1}$ In numerical simulations, we approximate the delta function in (4) as a Gaussian with narrow width-see section 5 for more details.
}

After dropping terms involving the third cumulant we can form the closed system for the evolution of the first and second cumulants of the flow:

$$
\begin{aligned}
\partial_{t} \bar{u} & =\mathscr{R}(C)-\bar{u}, \\
\partial_{t} C & =-\mathscr{L} C+\mathscr{N}(\bar{u}, C)+\varepsilon Q .
\end{aligned}
$$

The derivation of (5) has been presented many times; the reader is referred to, for example, the work by Farrell and Ioannou (2003), Srinivasan and Young (2012), and Bakas et al. (2015). In (5), $\mathscr{B}$ is the operator given in (A1) that governs the linear eddy dynamics, and $\mathscr{N}$ is the nonlinear operator given in (A2) that governs the interaction between the eddies and the instantaneous mean flow $\bar{u}(y, t)$. The mean flow $\bar{u}$ is driven by the ensemble mean eddy vorticity flux $\overline{v^{\prime} \zeta^{\prime}}$, which is expressed as a linear function of the eddy vorticity covariance $C$ through $\mathscr{B}(C)$ with $\mathscr{B}$ given in (A3).

The mean-flow energy density $E_{m}$ and the eddy energy density $E_{p}$ are

$$
\begin{aligned}
E_{m} & =\int_{\infty} d^{2} x \frac{1}{2} \bar{u}^{2}, \\
E_{p} & =\int_{\infty} d^{2} \mathbf{x} \frac{1}{2}\left[\overline{\mathbf{u}^{\prime}\left(\mathbf{x}_{a}\right) \cdot \mathbf{u}^{\prime}\left(\mathbf{x}_{b}\right)}\right]_{a=b} \\
& =-\int_{\infty} d^{2} \mathbf{x} \frac{1}{4}\left[\left(\Delta_{a}^{-1}+\Delta_{b}^{-1}\right) C\right]_{a=b},
\end{aligned}
$$

where $\int_{\infty} \stackrel{\text { def }}{=} \lim _{L \rightarrow \infty}(2 L)^{-2} \int_{-L}^{L} \int_{-L}^{L}$, the subscripts on the Laplacian indicate the specific variable the operator is acting on, and subscript $a=b$ implies that the function of $\mathbf{x}_{a}$ and $\mathbf{x}_{b}$, for example, inside the square brackets on the right-hand side of (6), is transformed into a function of a single variable by setting $\mathbf{x}_{a}=\mathbf{x}_{b}=\mathbf{x}$. The total averaged energy density relaxes over the dissipation scale [which is of $O(1)$ in the nondimensional equations] to the energy supported under stochastic forcing and dissipation:

$$
E(t) \stackrel{\text { def }}{=} E_{m}(t)+E_{p}(t)=\left[E(0)-\frac{\varepsilon}{2}\right] e^{-2 t}+\frac{\varepsilon}{2} .
$$

Therefore, the total energy remains bounded under S3T dynamics (Bakas and Ioannou 2019a).

\section{The flow-forming instability and the underlying eddy-mean flow dynamics}

S3T dynamics, (5), under homogeneous stochastic forcing admit, for all parameter values, a homogeneous equilibrium with zero mean flow and homogeneous eddy covariance: 


$$
\bar{u}^{e}=0, \quad C^{e}\left(\mathbf{x}_{a}-\mathbf{x}_{b}\right)=\frac{\varepsilon}{2} Q\left(\mathbf{x}_{a}-\mathbf{x}_{b}\right) .
$$

The homogeneous equilibrium state, $(8)$, becomes unstable at certain parameter values and bifurcates to inhomogeneous equilibria, a class of which are zonal jets. The stability of the homogeneous state, (8), is addressed by linearizing (5) around (8). Since (8) is homogeneous, the eigenfunctions consist of a sinusoidal mean-flow perturbation $\delta \bar{u} e^{\sigma t}$ and a perturbation covariance $\delta C e^{\sigma t}$ with a sinusoidal inhomogeneous part:

$$
\delta \bar{u}=e^{i n y}, \quad \delta C=\tilde{C}_{n}^{(h)}\left(\mathbf{x}_{a}-\mathbf{x}_{b}\right) e^{i n\left(y_{a}+y_{b}\right) / 2},
$$

where $n$ is a real wavenumber that indicates the length scale of the jets. The corresponding eigenvalues $\sigma$ satisfy (see appendix A)

$$
\sigma+1=f\left(\sigma \mid \delta \bar{u}, C^{e}\right)=\varepsilon f(\sigma \mid \delta \bar{u}, Q / 2),
$$

where $f$ is the vorticity flux induced by the distortion of the eddy equilibrium field $C^{e}$ by the mean flow $\delta \bar{u}$; the expression for $f$ is given in (A9). This induced vorticity flux is referred to as the vorticity flux feedback on $\delta \bar{u}$. For the ring forcing considered in this study, the fastest-growing instability for $n<1$ has a real eigenvalue $\sigma$, and therefore, the emergent jets are not translating in the $y$ direction. The vorticity flux feedback at marginal stability,

$$
f_{r} \stackrel{\text { def }}{=} \operatorname{Re}[f(\sigma=0 \mid \delta \bar{u}, Q / 2)],
$$

which is positive in this case, has the tendency to reinforce the preexisting jet perturbation $\delta \bar{u}$ and therefore destabilizes it. With dissipation, the critical parameter $\varepsilon$ at which the homogeneous equilibrium becomes unstable to a jet with wavenumber $n$ is $\varepsilon_{t}(n)=1 / f_{r}$, and for all values of $\beta$, there is a minimum energy input rate,

$$
\varepsilon_{c} \stackrel{\text { def }}{=} \min _{n}\left[\varepsilon_{t}(n)\right],
$$

above which the homogeneous state is unstable and jet formation occurs.

It is instructive to identify which wave components (of the incoherent flow) contribute to the instability process. For the forcing spectrum (4) we may express the vorticity flux feedback at the stability boundary $(\sigma=0)$ as

$$
f_{r}=\int_{0}^{\pi / 2} \mathscr{F}(\vartheta, n) d \vartheta
$$

where $\mathscr{F}(\vartheta, n)$ is the contribution to $f_{r}$ from the four wave components with wavevectors $\mathbf{k}= \pm(\cos \vartheta, \sin \vartheta)$ when the homogeneous equilibrium is perturbed by a jet perturbation with wavenumber $n$. Angle $\vartheta$ measures the inclination of the wave phase lines with respect to the $y$ axis. The precise expression for $\mathscr{F}(\vartheta, n)$ is given in (A12). Positive values of $\mathscr{F}$ indicate that waves with phase lines inclined at angle $|\vartheta|$ produce upgradient vorticity fluxes that are destabilizing the jet perturbation $n$. In general, destabilizing vorticity fluxes are produced by waves with phase lines closely aligned to the $y$ axis (with small $\left|k_{y}\right|$ ) as shown in Fig. 2.

Figure 3 shows the contribution $\mathscr{F}(\vartheta, n)$ as a function of $\vartheta$ for the most unstable jet $n_{c}$ for the cases with $\beta=0.1$ and $\beta=100$. When $\beta \ll 1, \mathscr{F}(\vartheta, n)$ is positive for angles satisfying $4 \sin ^{2} \vartheta<1+n^{2}$. This condition is derived for $\beta=0$ but is also quite accurate for small $\beta$, as shown in Fig. 3a (Bakas et al. 2015). The contribution from all angles is small (of order $\beta^{2}$ ), as the positive contribution at small angles is compensated by the negative contribution at larger angles. For $\beta \gg 1$, only waves with phase lines almost parallel to the $y$ axis $\left(\left|k_{y}\right| \approx 0\right)$ contribute significantly to the vorticity fluxes (see Fig. 3). When integrated over all angles, the resulting vorticity flux feedback is positive and $O\left(\beta^{-2}\right)$. The wave-mean flow dynamics underlying these contributions at all values of $\beta$ can be understood by considering the evolution of wave groups in the sinusoidal flow and were studied in detail by Bakas and Ioannou (2013b).

\section{The G-L dynamics governing the nonlinear evolution of the flow-forming instability}

In this section we discuss how the equilibration of the zonal jet instabilities is achieved for energy input rates just above the critical threshold $\varepsilon_{c}$. As it will be seen, the weak zonal jet equilibria are established through the equilibration of the most unstable eigenfunction with wavenumber $n_{c}$ as a nonlinear feedback modulates the eddy covariance in order to conserve energy and forms jet structures at the second harmonic $2 n_{c}$. It is through this energy conservation feedback along with the interaction with the $2 n_{c}$ jet that equilibration is achieved.

To derive the asymptotic dynamics that govern the evolution of the jet amplitude we perform a multiple-scale perturbation analysis of the nonlinear dynamics near the marginal point. Before proceeding with the multiple-scale analysis we present an intuitive argument that suggests the appropriate slow time and slow meridional spatial scales.

\section{a. The appropriate slow length scale and slow time scale}

For a stochastic excitation with energy input rate $\varepsilon=\varepsilon_{c}$, zonal jets with wavenumber $n=n_{c}$ are marginally stable. If the energy input rate is slightly supercritical, 

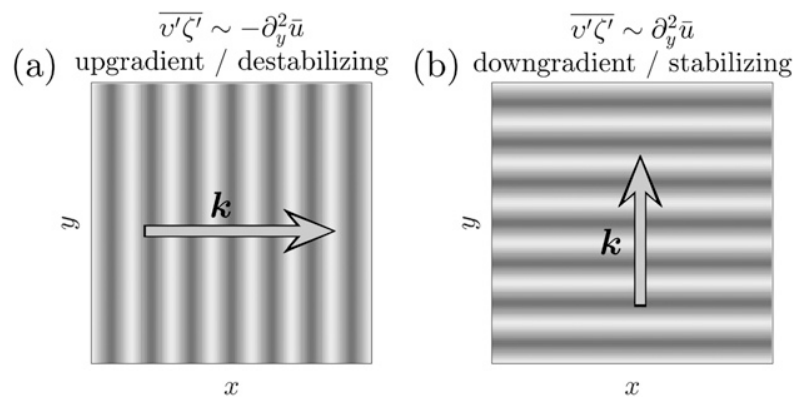

FIG. 2. (a) Waves with small $\left|k_{y}\right|$ produce upgradient vorticity fluxes that destabilize any mean-flow perturbation superimposed on the homogeneous turbulent equilibrium; (b) waves with large $\left|k_{y}\right|$ produce downgradient vorticity fluxes that tend to diminish mean-flow perturbations.

$$
\varepsilon=\varepsilon_{c}\left(1+\mu^{2}\right)
$$

with $\mu \ll 1$ a parameter that measures the supercriticality, then zonal jets with wavenumbers $\left|n-n_{c}\right|=$ $O(\mu)$ are unstable and grow at a rate of $O\left(\mu^{2}\right)$. To see this expand the eigenvalue relation (10) near $\varepsilon_{c}$,

$$
\begin{aligned}
\sigma= & \mu^{2} \varepsilon_{c} f_{r}+\varepsilon_{c}\left(\frac{\partial f}{\partial \sigma}\right)_{c} \sigma+\frac{\varepsilon_{c}}{2}\left(\frac{\partial^{2} f}{\partial n^{2}}\right)_{c}\left(n-n_{c}\right)^{2} \\
& +O\left[\sigma^{2},\left(n-n_{c}\right)^{3}\right],
\end{aligned}
$$

where the subscript $c$ denotes that the derivatives are evaluated at the threshold point $(\sigma, \mu, n)=\left(0,0, n_{c}\right)$.

Exactly at the minimum threshold, the function $f$ has a maximum at $n=n_{c}\left[(\partial f / \partial n)_{c}=0\right.$ and $\left.\left(\partial^{2} f / \partial n^{2}\right)_{c}<0\right]$ with value $\varepsilon_{c} f_{r}=1$, which as seen from (10) implies that $\sigma=0$. Thus the approximate eigenvalue relation in (15) predicts that the locus of points of marginal stability $(\sigma=0)$ on the $\varepsilon-n$ plane lie on the parabola:

$$
\left(n-n_{c}\right)^{2}=\frac{2}{\left|f_{c}^{\prime \prime}\right|}\left(\varepsilon / \varepsilon_{c}-1\right)=\frac{2 \mu^{2}}{\left|f_{c}^{\prime \prime}\right|}
$$

where $\left|f_{c}^{\prime \prime}\right| \stackrel{\text { def }}{=} \partial^{2} f /\left.\partial n^{2}\right|_{c}$.

Using (15) we can estimate the growth rate $\sigma$ at supercriticality $\mu$. We find that jets with wavenumber $n=n_{c}+\mu \nu$ grow approximately at rate

$$
\sigma=\mu^{2}\left(1-c_{2} \nu^{2}\right) / c_{1},
$$

with

$$
c_{1} \stackrel{\text { def }}{=} 1-\varepsilon_{c}\left(\frac{\partial f}{\partial \sigma}\right)_{c} \quad \text { and } \quad c_{2} \stackrel{\text { def }}{=} \frac{\varepsilon_{c}}{2}\left|f_{c}^{\prime \prime}\right| .
$$

The analytic expressions for $c_{1}$ and $c_{2}$ are given in (B13) and (B20). Coefficient $c_{1}$ is positive for stochastic excitations with spectrum (4). From (17), we deduce that for any $\mu$ only jets with
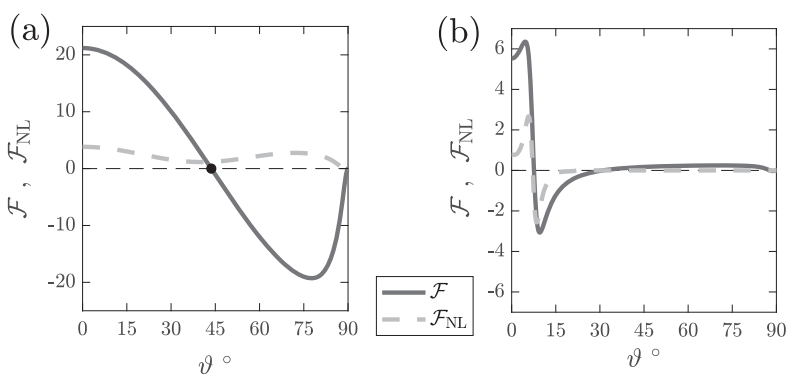

FIG. 3. The contribution $\mathscr{F}$ to the vorticity flux feedback $f_{r}$ for the most unstable jet eigenfunction from the waves with phase lines inclined at angle $\vartheta$ with respect to the meridional (solid curves). (a) The case with $\beta=0.1$; (b) $\beta=100$. In (a), the angle $\vartheta=$ $\arcsin \left[(1 / 2)\left(1+n^{2}\right)^{1 / 2}\right]$ that separates the waves with positive (destabilizing) and negative (stabilizing) contribution to the vorticity flux feedback for $\beta=0$ is indicated with the filled circle. Also, dashed curves show the contribution $\mathscr{F}_{\mathrm{NL}}$ to the nonlinear Landau coefficient $c_{3}$ for the most unstable jet eigenfunction as a function of the wave angle $\vartheta$ (see section 6 ).

$$
|\nu|<\nu_{e} \stackrel{\text { def }}{=} 1 / \sqrt{c_{2}}
$$

can become unstable.

Equations (16) and (17) establish the initial assertion: for $\mu \ll 1$, zonal jets with wavenumbers $\left|n-n_{c}\right|=O(\mu)$ grow at a rate $\sigma=O\left(\mu^{2}\right)$.

The validity of the approximate eigenvalue relation (17) as a function of supercriticality $\mu$ is shown in Figs. $4 \mathrm{a}$ and $4 \mathrm{~b}$. By comparing the exact growth rates as given by (10) and the growth rates obtained from the approximation (17), we see that the approximate eigenvalue dispersion may not be as accurate in three ways: predicting the maximum growth rate, predicting the wavenumber at which maximum growth occurs, and predicting the asymmetry of the exact growth rates about the maximal wavenumber. These three differences are indicated by the arrows in Figs. $4 \mathrm{a}$ and $4 \mathrm{~b}$ and are quantified in Figs. 4c-e. Figure 4c compares the exact wavenumber of maximum growth $n_{\max }$ to the critical wavenumber $n_{c}$ assumed by approximation (17). We see that $n_{\max }$ is very close to $n_{c}$ up to $\mu \approx 1$ with the error growing as $\mu^{2}$. This is in agreement with the error in (15) being of $O\left(\sigma^{2}\right)$. In addition, the exact growth rate $\sigma\left(n_{c}\right)$ is very close to $\mu^{2} / c_{1}$, as shown in Fig. $4 \mathrm{~d}$ for $\mu$ up to $O(1)$, the growth rate being overestimated by (17) for higher values. Finally, the parabolic approximation (17) to the growth rates predicts that the wavenumbers $\pm \nu_{e}$ are marginally stable $\left[\sigma\left( \pm \nu_{e}\right)=0\right]$. Figure $4 \mathrm{e}$ shows the exact growth rates at $\pm \nu_{e}$ at $\mu=0.5$; these are far from zero for both low and high values of $\beta$. The parabolic approximation works best for intermediate $\beta$ values, that is, for $\beta=O(1)$. To summarize, the approximated maximum growth rate $\mu^{2} / c_{1}$ as well as the critical 

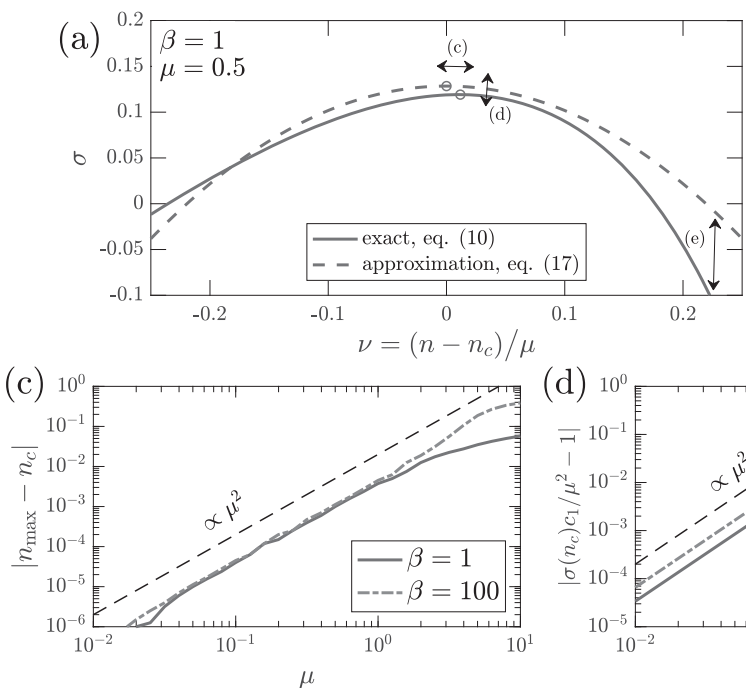

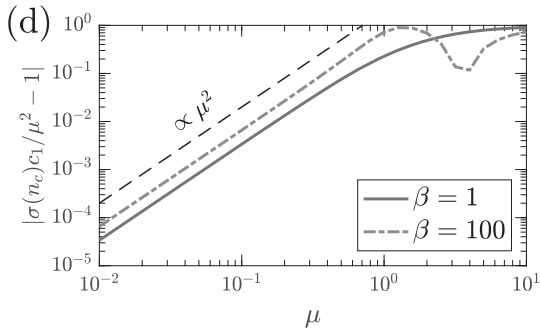

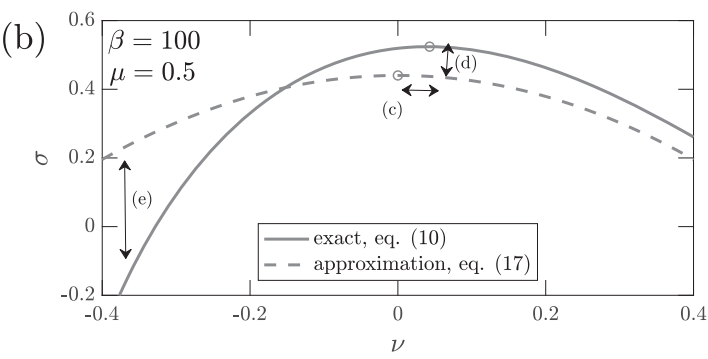

(e)

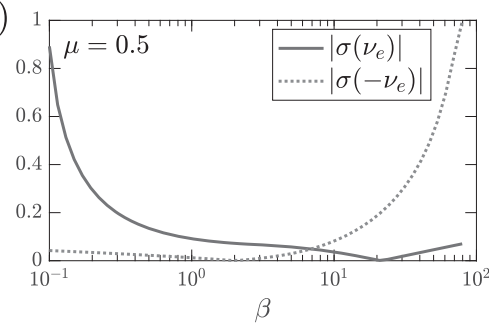

FIG. 4. Validity of the approximate eigenvalue relation, (17). (a) Comparison of the growth rates for jet perturbations with wavenumber $\nu$ as predicted by the exact eigenvalue relation (10) (solid curve) and by the parabolic approximation, (17) (dashed curve), for supercriticality $\mu=0.5$ and $\beta=1$. Circles mark the maximum growth rate: for (10) this is at wavenumber $n_{\max }$, while for (17) it is at $n_{c}$. (b) As in (a), but for $\beta=100$. (c) The difference between the exact wavenumber of maximum growth $n_{\max }$ and the approximate wavenumber of maximum growth $n_{c}$ as a function of the supercriticality $\mu$. (d) The relative difference between the exact growth rate $\sigma$ for a jet at wavenumber $n_{c}$ and the approximate growth rate $\mu^{2} / c_{1}$ as a function of supercriticality $\mu$. (e) The exact growth rate of jet perturbations with wavenumbers $\nu_{e}$ and $-\nu_{e}$ as a function of $\beta$ for supercriticality $\mu=0.5$. The parabolic approximation predicts zero growth for these marginal wavenumbers.

wavenumber $n_{c}$ that achieves this maximum growth are both good approximations for supercriticalities up to $\mu=O(1)$; the parabolic dependence of growth rate for wavenumbers away from $n_{c}$ is a good approximation at $\mu>0.1$ only for intermediate values of $\beta$. As it will be seen, this has implications on the validity of the weakly nonlinear dynamics derived next.

\section{b. G-L dynamics for weakly supercritical zonal jets}

Since the excess energy available for flow formation is of order $\mu^{2} \varepsilon_{c}$, we expect intuitively the mean-flow amplitude to be of order $\mu$. Therefore, to obtain the dynamics that govern weakly supercritical zonal flows, we expand the mean flow $\bar{u}$ and the covariance $C$ of the S3T equations, (5), as

$$
\begin{aligned}
\bar{u}= & \mu \bar{u}_{1}(y, Y, T)+\mu^{2} \bar{u}_{2}(y, Y, T)+O\left(\mu^{3}\right), \\
C= & C^{e}\left(\mathbf{x}_{a}-\mathbf{x}_{b}\right)+\mu C_{1}\left(\mathbf{x}_{a}, \mathbf{x}_{b}, Y_{a}, Y_{b}, T\right) \\
& +\mu^{2} C_{2}\left(\mathbf{x}_{a}, \mathbf{x}_{b}, Y_{a}, Y_{b}, T\right)+O\left(\mu^{3}\right),
\end{aligned}
$$

Guided by (16) and (17), we have assumed that the zonal jet and its associated covariance evolve from the marginal values at the slow time scale $T \stackrel{\text { def }}{=} \mu^{2} t$ while being modulated at the long meridional scale $Y \stackrel{\text { def }}{=} \mu y$.

Details of the perturbation analysis are given in appendix B; here we present the backbone. We introduce (20) in (5) and gather terms with the same power of $\mu$. At leading-order $\mu^{0}$, we recover the homogeneous equilibrium (8). At order $\mu^{1}$, the emergent zonal jet and the covariance are the modulated S3T eigenfunction:

$$
\begin{aligned}
\bar{u}_{1}= & A(Y, T) e^{i n_{c} y}+\text { c.c. } \\
C_{1}= & {\left[A\left(Y_{a}, T\right) G^{+}\left(0 \mid \mathbf{x}_{a}-\mathbf{x}_{b}\right)\right.} \\
& \left.-A\left(Y_{b}, T\right) G^{-}\left(0 \mid \mathbf{x}_{a}-\mathbf{x}_{b}\right)\right] e^{i n_{c}\left(y_{a}+y_{b}\right) / 2}+\text { c.c. },
\end{aligned}
$$

with $G_{c}^{ \pm}$defined in (A8) and evaluated at $n=n_{c}$.

Having determined $C_{1}$ we proceed to determine the order $\mu^{2}$ correction of the covariance $C_{2}$. This step of the calculation is facilitated if we disregard the dependence on the slow spatial scale $Y$ in the amplitude $A$, as well as that in $C_{1}$ and $C_{2}$. Parker and Krommes (2014) showed that the nonlinear term of the asymptotic dynamics responsible for the equilibration of the amplitude $A$ can be obtained using this simplification, while the contribution to the asymptotic dynamics from the slow varying latitude $Y$ is the addition of a diffusion term with the diffusion coefficient $c_{2}$ in (18). At order $\mu^{2}$ a zonal jet with wavenumber $2 n_{c}$ emerges:

$$
\bar{u}_{2}=\alpha_{2} A(T)^{2} e^{2 i n_{c} y}+\text { c.c. },
$$


where $\alpha_{2}$ is given in (B7) and for the forcing considered is negative $\left(\alpha_{2}<0\right)$. The associated covariance at order $\mu^{2}$,

$$
\begin{aligned}
C_{2}= & C^{e}\left(\mathbf{x}_{a}-\mathbf{x}_{b}\right)+C_{20}\left(\mathbf{x}_{a}-\mathbf{x}_{b}\right) \\
& +C_{22}\left(\mathbf{x}_{a}-\mathbf{x}_{b}\right) e^{2 i n_{c}\left(y_{a}+y_{b}\right) / 2}+\text { c.c. },
\end{aligned}
$$

consists of the homogeneous part, $C^{e}+C_{20}$, and also an inhomogeneous contribution at wavenumber $2 n_{c}$. (Note that, as implied by (14), the forcing covariance $Q$ appears both at order $\mu^{0}$ and at order $\mu^{2}$.)

The homogeneous covariance contribution, $C^{e}+C_{20}$, is required at order $\mu^{2}$ so that the energy conservation, (7), is satisfied. To show this, note that, as the instability develops on a slow time scale, the total energy density has already assumed (over an order-one time scale) its steady state value $\varepsilon / 2$ [see (7)], and therefore, the mean-flow energy growth must be accompanied by a decrease in the eddy energy. This decrease is facilitated by a concomitant change of the eddy covariance at order $\mu^{2}$. Specifically, by introducing the perturbation expansion, (20), in (7) at steady state, we obtain at leading order $\mu^{0}$ the trivial balance:

$$
-\int_{\infty} d^{2} \mathbf{x} \frac{1}{4}\left[\left(\Delta_{a}^{-1}+\Delta_{b}^{-1}\right) C^{e}\right]_{a=b}=\frac{\varepsilon_{c}}{2} .
$$

At order $\mu^{1}$ the eddy covariance does not contribute to the energy since $C_{1}$ is harmonic in $y$ and integrates to zero:

$$
\int_{\infty} d^{2} \mathbf{x} \frac{1}{4}\left[\left(\Delta_{a}^{-1}+\Delta_{b}^{-1}\right) C_{1}\right]_{a=b}=0 .
$$

At order $\mu^{2}$ we use (i) (23) and (ii) the fact that the inhomogeneous component $C_{22} e^{2 i n_{c}\left(y_{a}+y_{b}\right) / 2}$ is harmonic and integrates to zero to obtain

$$
\int_{\infty} d^{2} \mathbf{x} \frac{1}{4}\left[\left(\Delta_{a}^{-1}+\Delta_{b}^{-1}\right) C_{20}\right]_{a=b}=\int_{\infty} d^{2} \mathbf{x} \frac{1}{2} \bar{u}_{1}^{2} .
$$

Thus the homogeneous deviation from the equilibrium covariance must produce a perturbation energy defect to counterbalance the energy growth of the mean flow. We refer to $C_{20}$ as the eddy energy correction term. However, we note that the correction to the homogeneous part of the covariance does not only change the mean eddy energy but also other eddy characteristics, such as the mean eddy anisotropy, that also might play a role in the equilibration process.

At order $\mu^{3}$ secular terms appear that, if suppressed, yield an asymptotic perturbation expansion up to time $O\left(1 / \mu^{2}\right)$. Suppression of these secular terms requires that the amplitude $A$ of the most unstable jet with wavenumber $n_{c}$ satisfies

$$
c_{1} \partial_{T} A=A-c_{3} A|A|^{2} .
$$

If we now allow the amplitude to also evolve with the slow-scale $Y$ and add the diffusion term $c_{2} \partial_{Y}^{2} A$ on the right-hand side of (26), we obtain the celebrated real G-L equation:

$$
c_{1} \partial_{T} A=A+c_{2} \partial_{Y}^{2} A-c_{3} A|A|^{2} .
$$

For forcing with spectrum (4) all three coefficients $c_{1}, c_{2}$, and $c_{3}$ are real and positive. The coefficients $c_{1}$ and $c_{2}$ are the coefficients in the Taylor expansion, (15), and are given in (18).

The G-L equation, (27), has a steady solution $A=0$. This solution is linearly unstable to modal perturbations $e^{i \nu Y+\sigma T}$, with growth rate $\mu^{2}\left(1-\nu^{2} c_{2}\right) / c_{1}$; the most unstable mode occurs at $\nu=0$. This is the flow-forming SSD instability of the homogeneous equilibrium state in the G-L framework [cf. (17)]. The G-L equation has also the nonlinear harmonic equilibria:

$$
\begin{aligned}
& A(Y)=R_{0}(\nu) e^{i(\nu Y+\phi)} \text { with } \\
& R_{0}(\nu)=\sqrt{\left(1-\nu^{2} c_{2}\right) / c_{3}},
\end{aligned}
$$

and $\varphi$ an undetermined phase that reflects the translational invariance of the system in $y$. These equilibria are the possible finite-amplitude jets that emerge at low supercriticality. However, as will be shown in the next section, some of these equilibria are susceptible to a secondary SSD instability and evolve through jet merging or jet branching to the subset of the stable attracting states.

The $\nu=0$ jet is the state with the largest amplitude,

$$
R_{0}(0)=1 / \sqrt{c_{3}} .
$$

\section{Comparison of the predictions of $G-L$ dynamics with S3T dynamics for the equilibrated jets}

In this section we test the validity of the weakly nonlinear $\mathrm{G}-\mathrm{L}$ dynamics by comparing its predictions for the amplitude of the equilibrated jets with fully nonlinear S3T dynamics. We consider the S3T dynamical system, (5), in a doubly periodic domain $2 \pi L_{*} \times 2 \pi L_{*}$ with a $128^{2}$ grid resolution and $L_{*}=1$, as well as the G-L dynamics with periodic boundary conditions for the amplitude of the jet $A$ on the same domain. We approximate the delta function in the ring forcing, (4), as

$$
\delta\left(k_{*}-k_{f *}\right) \mapsto \frac{e^{-\left(k_{*}-k_{f^{*}}\right)^{2} /\left(2 \delta_{f^{*}}^{2}\right)}}{\sqrt{2 \pi} \delta_{f^{*}}},
$$


with $k_{x^{*}} L_{*}$ and $k_{y^{*}} L_{*}$ assuming integer values. [The asterisks denote dimensional values, as in, e.g., (1).] Forcing (30) injects energy in a narrow ring in wavenumber space with radius $k_{f *} L_{*}=10$ and width $\delta_{f *} L_{*}=1.5$. We note that even though (30) is a good approximation of the delta-ring forcing, (4), small quantitative differences are to be expected. For example, the critical energy input rates for jet emergence obtained from the discrete finite-ring excitation differ by as much as $4 \%$ from the corresponding values obtained from the delta-ring forcing, (4). Since the equilibrated jet amplitudes are of order $\mu \ll 1$, we use the exact values for the critical energy input rates obtained for the discrete finite-ring excitation.

We also consider $r_{*}=0.1$ and vary $\beta_{*}$ as well as the energy input rate $\varepsilon_{*}$, that is, the bifurcation parameter. The eigenvalue relation for the flow-forming instability is obtained by substituting the integrals in (10) with sums over the allowed wavenumbers. However, the comparison with the predictions of the G-L dynamics with periodic boundary conditions in the meridional is trickier. Because of the periodic boundary conditions in the jet amplitude, a harmonic mean flow $A(Y, T)=e^{i \nu Y}$ with wavenumber $\nu$ is realizable within our domain only if its dimensional wavenumber $n_{*}=\left(\mu \nu+n_{c}\right) k_{f}$ is an integer. Therefore, we carefully pick $\beta_{*}$ so that the marginal wavenumber $n_{c} k_{f} *$ always assumes an integer value; for $k_{f^{*}}=10 / L_{*}$ this leaves us with nine possible values for $\beta_{*}$ covering the range $3 \times 10^{-1}<\beta_{*}<2 \times 10^{3}$. The lowest and highest marginal $\beta_{*}$ values yield marginal jets at the lowest- and highest-allowed wavenumber possible within our domain; $1 / L_{*}$ and $k_{f *}-1 / L_{*}$, respectively. We excluded these values for $\beta_{*}$, since they do not allow us to study the finite-amplitude stability of side-band jets (i.e., jets at larger or smaller scale compared to the scale of $n_{c^{*}}$ ). Therefore, in our comparisons, we use only the remaining seven allowed values of $\beta_{*}$, which are shown in Table 1 .

We calculate the finite-amplitude equilibrated jets of the nonlinear S3T dynamical system, (5), using Newton's method with the initial guess provided by (29). ${ }^{2}$ All jet equilibria we compute in this section are hydrodynamically stable. At small supercriticalities, the jet amplitude is small, and the linear operator is dominated by dissipation. Thus, all instabilities we discuss here are SSD instabilities (see paragraph 3 in section 1).

\section{a. Equilibration of the most unstable jet $n_{c}$}

Consider first the most unstable jet perturbation with wavenumber $n_{c}$. Figure 5 shows the Fourier amplitude

\footnotetext{
${ }^{2}$ For details regarding Newton's root-finding algorithm for system (5), the reader is referred to appendix I in the thesis of Constantinou (2015).
}

TABLE 1. Exact values of nondimensional planetary vorticity gradient $\beta \stackrel{\text { def }}{=} \beta_{*} /\left(k_{f} * r_{*}\right)$ used in the S3T simulations of section 5 and their corresponding values of the dimensional critical wavenumber $n_{c *}$.

\begin{tabular}{ccc}
\hline \hline Notation & $\beta$ & $n_{c} *$ \\
\hline$\beta_{1}$ & 1.1915 & 8 \\
$\beta_{3}$ & 3.0235 & 7 \\
$\beta_{6}$ & 6.2761 & 6 \\
$\beta_{12}$ & 12.136 & 5 \\
$\beta_{24}$ & 24.576 & 4 \\
$\beta_{58}$ & 58.137 & 3 \\
$\beta_{192}$ & 192.62 & 2 \\
\hline
\end{tabular}

of the equilibrated jet dominated by wavenumber $n_{c}$ for four values of $\beta$. We see that, for $\beta \lesssim 12$, the amplitude is given, to a very good approximation, by (29) for supercriticality up to $\mu \approx 0.2$ (see Figs. 5a,b). For larger supercriticality, the amplitude of the equilibrated jet is not well captured by (29); the jet amplitude is overestimated for $\beta \lesssim 12$ while it is underestimated for $\beta \gtrsim 12$. We note here that S3T equilibria with dominant wavenumber $n_{c}$ (as predicted by the G-L dynamics) exist at even larger supercriticalities, but these were found to be S3T unstable.

Surprisingly, for $\beta \gtrsim 20$, there exist multiple equilibria for the same supercriticality $\mu$ (see Figs. $5 \mathrm{c}, \mathrm{d}$ ). Specifically, there exists a branch of stable equilibria apart from the jets connected to the homogeneous equilibrium (cf. triangles in Figs. $5 \mathrm{c}, \mathrm{d}$ vs the circles). For $\mu \gtrsim 0.2$, the lowerbranch equilibria, predicted by the G-L dynamics, cease to exist; an infinitesimal harmonic jet perturbation with wavenumber $n_{c}$ ends up in the upper branch. Equally interesting is the fact that the upper branch extends to subcritical values of the energy input rate with respect to the flow-forming instability of the homogeneous state, that is, for $\varepsilon<\varepsilon_{c}$. This is shown in Fig. 6a for $\beta_{58}$; similar subcritical jet equilibria were found for $\beta_{24}$ and $\beta_{192}$ (not shown). Thus, apart from the linear instability forming jets that has been extensively studied in the literature, there is a nonlinear instability for jet formation, the details of which will be discussed in section $6 \mathrm{~b}$. Since both the upper and the lower branches exist for a limited range of energy input rates, there is a hysteresis loop shown in Fig. 6a, with the dynamics landing on the upper or the lower branch of jet equilibria as $\varepsilon$ is varied. The two stable branches are connected with a branch of unstable equilibria (open circles) that were also found using Newton's method.

The jets on the lower and the upper stable branches are qualitatively different. Figures $6 \mathrm{~b}-\mathrm{e}$ compare the jet structure and spectra of two such equilibria in the case of $\beta_{58}$ and $\mu=0.05$. While the lower-branch jet consists mainly of $n_{c}$ and its double-harmonic $2 n_{c}$ with a much 

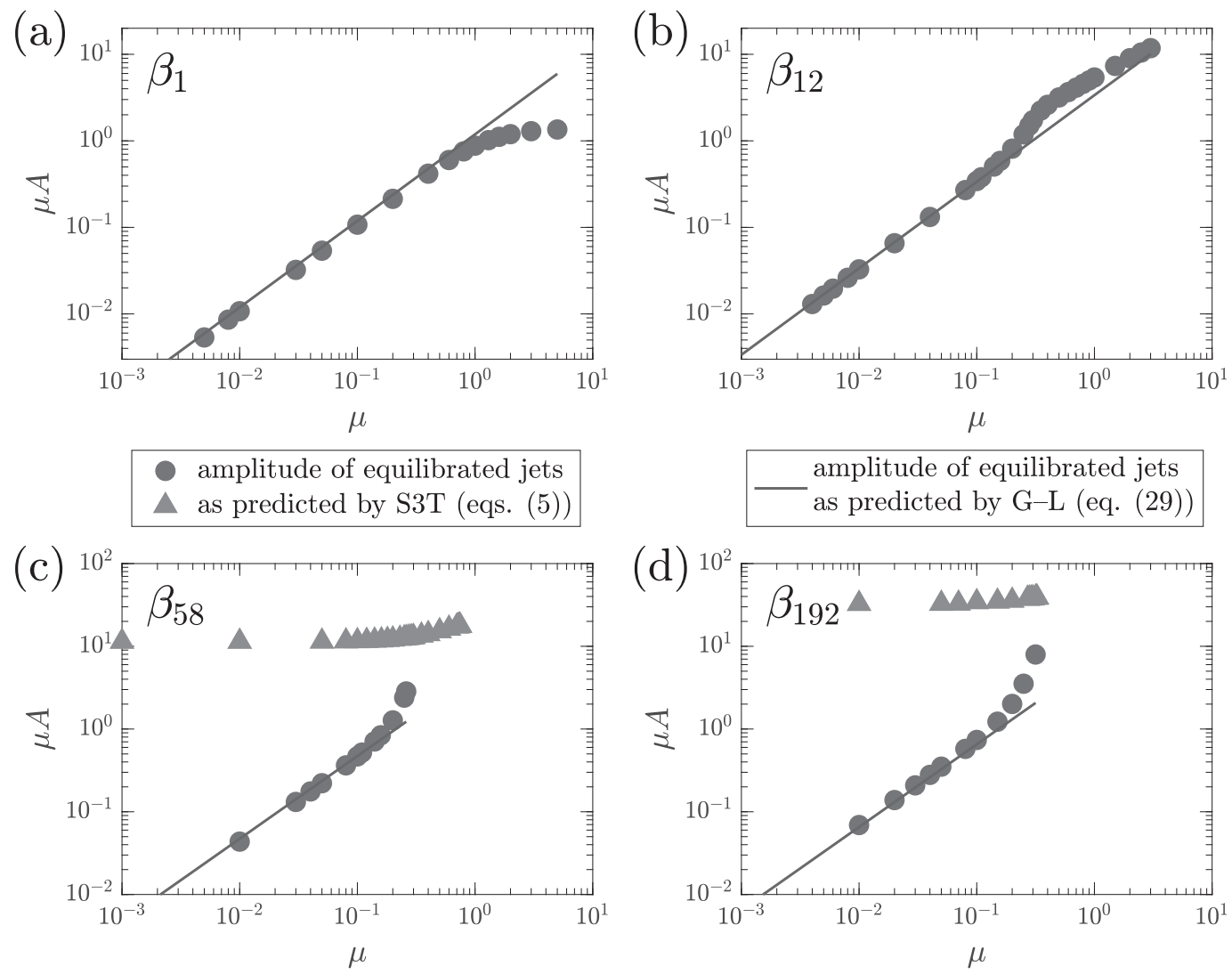

FIG. 5. The amplitude $\mu A$ of the equilibrated most unstable jet with wavenumber $n_{c}$ as a function of supercriticality $\mu$ for four values of $\beta$. The $\mathrm{G}-\mathrm{L}$ branch is shown with circles; the upper branch (which appears for $\beta \gtrsim 20$ ) is shown with triangles. Solid lines show the jet amplitude as predicted by the G-L [cf. (29)].

weaker Fourier amplitude in qualitative agreement with the G-L prediction of $\bar{u}_{2} \approx O\left(\mu^{2} A^{2}\right)$ [cf. (22a) and Fig. 6d], the upper-branch jet is stronger by two orders of magnitude, it contains more harmonics, and the Fourier amplitude of the double-harmonic $2 n_{c}$ is about half the amplitude of the leading harmonic $n_{c}$. As will be elaborated in section $6 \mathrm{~b}$, it is the interaction of the two Fourier components with wavenumbers $n_{c}$ and $2 n_{c}$ that supports the upper-branch equilibria.

\section{b. Equilibration of the side-band jets $n_{c} \pm 1 /\left(k_{f} L_{*}\right)$}

We now consider the jet equilibria that emerge from the equilibration of jet perturbations with wavenumbers close to $n_{c}$. While for an infinite domain there is a dense set of unstable jet perturbations with wavenumbers $\mu \nu$ close to $n_{c}$ [cf. (19)], for the doubly periodic box the first side-band jet instabilities have dimensional wavenumbers $n_{*}^{ \pm}=n_{c} k_{f} * \pm 1 / L_{*}$ or $\nu^{ \pm}= \pm 1 /\left(k_{f} * L_{*} \mu\right)$. Introducing $\nu^{ \pm}$in (19), we obtain that the parabolic approximation predicts that the homogeneous equilibrium becomes unstable to jet perturbations with wavenumber $\nu^{ \pm}$when $\mu_{\mathrm{GL}}>\sqrt{c_{2}} /\left(k_{f} * L_{*}\right)$. However, as shown in Fig. 4d, the parabolic approximation is not accurate, especially at low and large values of $\beta$. For example, for $\beta_{1}, \mu_{\mathrm{GL}}=0.4293$, while the exact dispersion relation predicts that jets with $\nu_{+}$and $\nu_{-}$are rendered neutral at $\mu_{\mathrm{ex}^{+}}=0.2140$ and $\mu_{\mathrm{ex}^{-}}=0.7953$, respectively. We therefore expect significant deviations from (28) for the amplitude of the equilibrated jets.

Figure 7 shows the equilibrated amplitude of the side-band jet perturbations with $\nu^{ \pm}$as a function of supercriticality for four values of $\beta$. While the functional dependence of the equilibrated amplitude on $\mu$ is qualitatively captured by (28) (dashed lines), there are significant quantitative differences, especially for $\beta_{1}$ and $\beta_{192}$. Since these quantitative differences are due to the failure of the parabolic approximation, a way to rectify them is to use in (28) an equivalent

$$
c_{2}^{\mathrm{ex} \pm} \stackrel{\text { def }}{=}\left(k_{f *} L_{*} \mu_{\mathrm{ex}^{ \pm}}\right)^{2},
$$

based on the supercriticality $\mu_{\mathrm{ex}}^{ \pm}$obtained from the exact dispersion relation, (10). The solid curves in Fig. 7 show the predicted amplitude using $c_{2}^{\mathrm{ex} \pm}$. We observe that, for 
(a)

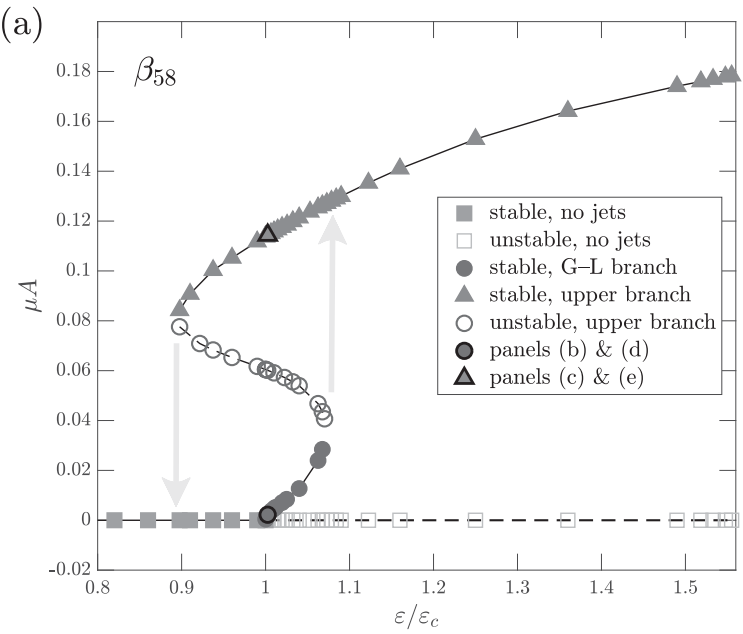

(b)

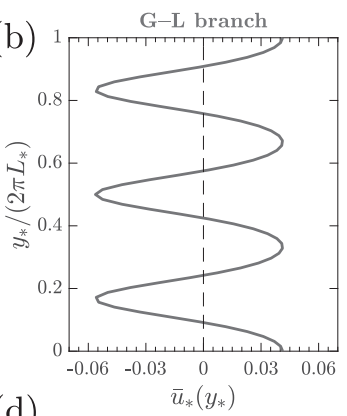

(d)

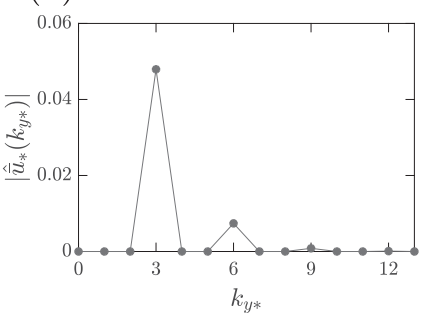

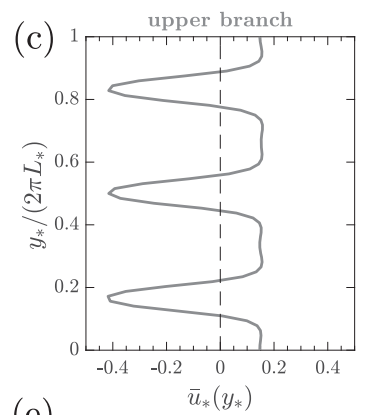

(e)

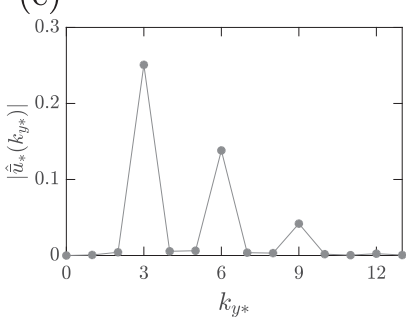

FIG. 6. The bifurcation diagram for $\beta_{58}$ (case shown in Fig. 5c). (a) The amplitude $\mu A$ of the equilibrated most unstable jet with wavenumber $n_{c}$ as a function of the energy input rate. Squares denote the homogeneous equilibrium, circles the lower branch predicted by the G-L dynamics, and the triangles the upper branch of equilibria. Open symbols denote unstable jet equilibria with respect to S3T dynamics; filled symbols denote stable jet equilibria. Multiple stable equilibria exist for $0.89 \leq \varepsilon / \varepsilon_{c} \leq 1.068$. (b)-(d) A comparison of the jet equilibrium structure and the jet spectra for $\varepsilon=1.0025$ (which corresponds to $\mu=0.05$ ) is shown. (b), (d) The lower G-L branch jet; (c),(e) the upper-branch jet. In (d), the amplitude prediction for $n_{c}$ by (29) and for $2 n_{c}$ by (22a) is also shown (open circles).

all values of $\beta$, the amplitude of the jets close to the bifurcation point is accurately predicted, and for the intermediate value of $\beta_{6}$, for which the exact dispersion is the closest to the parabolic profile, the agreement holds away from the bifurcation point as well. Finally, note that for large $\beta$ shown in Fig. $7 \mathrm{c}$ the additional upper branch of equilibria is found and this branch has the same characteristics as the upper branch of $n_{c}$ equilibria. That is, the equilibrated jets have a larger amplitude, and the Fourier amplitude of the double harmonic [in this case, it is the $2\left(n_{c}+1 / \mu k_{f} L_{*}\right)$ harmonic] is much larger compared to that for the equilibria in the $\mathrm{G}-\mathrm{L}$ branch.

Finally, we stress that the results in this section regarding the existence of the upper-branch equilibria as well as the accuracy of the G-L dynamics for the lower-branch equilibria are not quirks of the particular isotropic-forcing structure in (4). Similar qualitative behavior is found for forcing with anisotropic spectrum. Discussion regarding the effects of the structure of the forcing is found in appendix $\mathrm{C}$.

\section{The physical processes underlying the equilibration of the SSD instability of the homogeneous state}

One of the main objectives of this paper is to study the processes that control the halting of the flow-forming instability both for the low-branch equilibria, which are governed by the G-L dynamics, and for the upperbranch equilibria (cf. Figs. 5 and 6).

\section{a. Equilibration processes for the lower branch}

For G-L dynamics, the equilibration of the instability for the most unstable jet perturbation with wavenumber $n_{c}$ as well as for side-band jets (i.e., jets with scales close to $n_{c}$ ) is controlled by coefficient $c_{3}$ in the G-L equation, (27). We start with a discussion on how $c_{3}$, and consequently of the equilibration amplitude $R_{0}(0)$, depends on $\beta$; Fig. 8a shows the amplitude of the most unstable jet, $R_{0}(0)$, as a function of $\beta$. For $\beta \gg 1$ the emerging jets have large scales $\left(n_{c} \ll 1\right)$ and equilibrate at an amplitude that increases as $R_{0} \sim \beta^{1 / 3}$. For $\beta \ll 1$, the emerging jets have small scales $\left(n_{c} \approx 1\right)$, and their amplitude scales as $R_{0} \sim \beta^{2 / 3}$. The scaling of $R_{0}$ for $\beta \gg 1$ is found to be robust, that is, independent of the spectral properties of the forcing (cf. Fig. 8 and Fig. C1). On the other hand for $\beta \ll 1$ the amplitude $R_{0}$ depends crucially on the forcing structure; see appendix C. However, the regime $\beta \ll 1$ is uninteresting anyway since the anisotropy in the dynamics in (1) becomes vanishingly small and no zonal jets emerge.

The dependence of the amplitude $R_{0}(0)$ on $\beta$ can be understood by considering the contribution of the various wave components to $c_{3}$ in a similar manner as we did for $f_{r}$ in (13). Thus, we write 

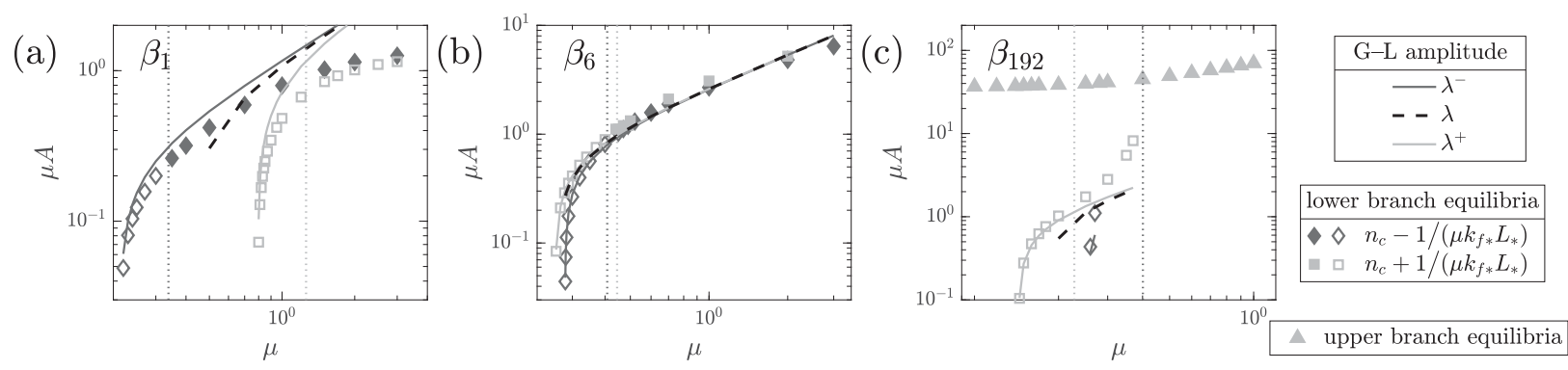

FIG. 7. The amplitude $\mu A$ of the equilibrated unstable jets with wavenumbers $n_{c}-1 /\left(\mu k_{f} L_{*}\right)$ (diamonds) and $n_{c}+1 /\left(\mu k_{f} * L_{*}\right)$ (squares) as a function of supercriticality $\mu$ for four values of $\beta$. The dashed lines show the amplitude predicted by the G-L dynamics [cf. (29)], while the solid lines show the amplitude predicted by the G-L dynamics with $c_{2}^{\text {ex }}$ as described in the text. Stable (unstable) equilibria are denoted with filled (empty) symbols, and the vertical dotted lines show the stability boundary, (58), obtained from the G-L dynamics (see section 7).

$$
c_{3}=\int_{0}^{\pi / 2} \mathscr{F}_{\mathrm{NL}}(\vartheta) d \vartheta
$$

where $\mathscr{F}_{\mathrm{NL}}$ is the contribution to $c_{3}$ from the four waves with wavevectors $\mathbf{k}= \pm(\cos \vartheta, \sin \vartheta)$. Figure 3 shows the contributions $\mathscr{F}_{\mathrm{NL}}(\vartheta)$ for two values of $\beta$.

For $\beta \ll 1$, all wave orientations contribute positively to $c_{3}$. As a result, the upgradient contributions to the vorticity flux feedback $\mathscr{F}$ at small $\vartheta$ are counteracted by $\mathscr{F}_{\text {NL}}$, while the downgradient contributions to $\mathscr{F}$ at higher $\vartheta$ are enhanced by $\mathscr{F}_{\mathrm{NL}}$. This leads to a rapid quenching of the instability and thus to a weak finite-amplitude jet.

For large $\beta, \mathscr{F}_{\mathrm{NL}}$ has roughly the same dipole structure centered about an angle $\vartheta_{0}$ as the vorticity flux feedback $\mathscr{F}$. Therefore, only waves with angles close to $\vartheta_{0}$ contribute appreciably to $c_{3}$. Waves with angles $|\vartheta|<\vartheta_{0}$ give positive contributions to $c_{3}$, while waves with angles $|\vartheta|>\vartheta_{0}$ give negative contributions to $c_{3}$. As a result, both the upgradient and the downgradient contributions to $\mathscr{F}$ are almost equally reduced, and the instability is only slowly hindered and thus allowed to drive jets to a much larger amplitude compared to $\beta \ll 1$. To understand the power-law increase of $R(0)$ with $\beta$, note that, as $\beta$ increases, (i) the heights of the dipole peaks grow linearly with $\beta$, (ii) the widths of the dipole peaks decrease as $\beta^{-2 / 3}$, and (iii) the structure of the dipole becomes more symmetric about $\vartheta_{0}$. Figure 9a demonstrates points i-iii. Thus, each of the positive and the negative contribution to $c_{3}$ scales as $\beta \times \beta^{-2 / 3}=\beta^{1 / 3}$, and their difference scales with the derivative, that is, as $d \beta^{1 / 3} / d \beta \propto \beta^{-2 / 3}$, leading to the increase of $R(0)$ with $\beta$ as $\beta^{1 / 3}$.

Next we investigate how each of the forced waves contribute in sustaining the equilibrated state of the most unstable jet $(\nu=0)$ with amplitude $R_{0}(0)$ by decomposing the portion of the vorticity flux exceeding dissipation, which is the sum of $f_{r}$ and $-c_{3} R_{0}(0)^{2}$, into contributions from various wave angles:

$$
f_{r}-c_{3} R_{0}(0)^{2}=\int_{0}^{\pi / 2}\left[\mathscr{F}(\vartheta)-R_{0}^{2} \mathscr{F}_{\mathrm{NL}}(\vartheta)\right] d \vartheta .
$$

Figure $9 \mathrm{~b}$ shows these contributions for three values of $\beta$. For small values of $\beta$, waves with angles $|\vartheta|<\pi / 4$ that drive the instability through their upgradient contribution also support the equilibrated jet. However, for $\beta \gg 1$, this picture is reversed. The instability is driven by waves with $|\vartheta|<\vartheta_{0}$ (mainly from waves with $|\vartheta| \approx 0$ )

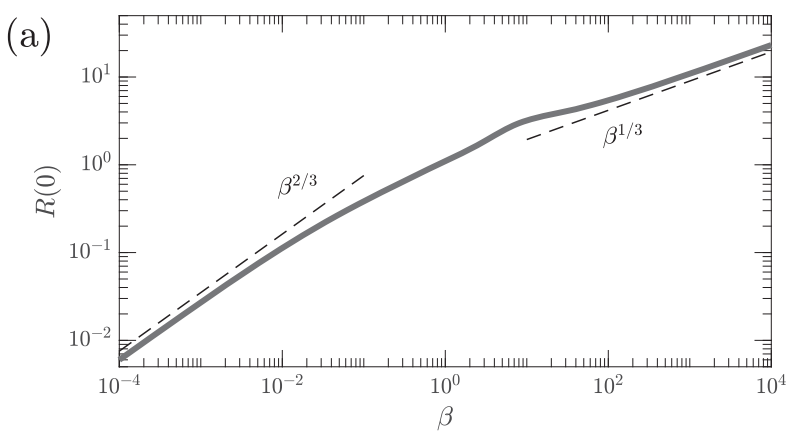

(b)

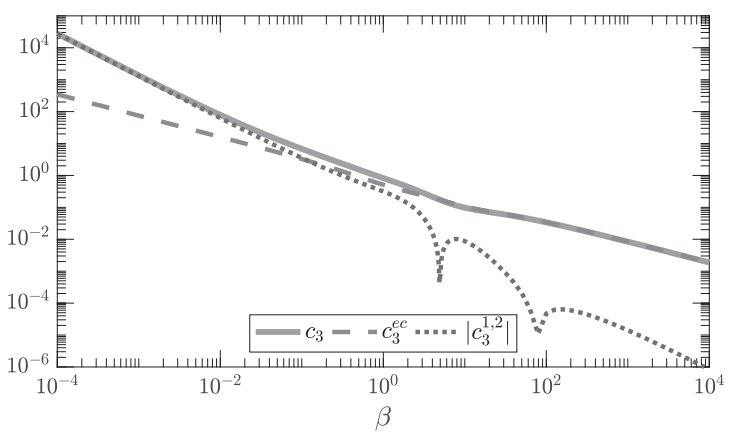

FIG. 8. (a) The amplitude $R_{0}(0)=1 / \sqrt{c_{3}}$ of the equilibrated most unstable jet with wavenumber $n_{c}$ as a function of $\beta$. Dashed lines show the $\beta^{1 / 3}$ and $\beta^{2 / 3}$ slopes for reference. (b) The coefficient $c_{3}$ and its decomposition into the contributions $c_{3}^{\text {ec }}$ and $c_{3}^{1,2}$ as a function of $\beta$. Coefficient $c_{3}^{1,2}$ is negative for $4.9 \lesssim \beta \lesssim 79$. However, for these values, $c_{3}^{1,2}$ are at least an order of magnitude less than $c_{3}^{\mathrm{ec}}$ and, therefore, are negligible. 


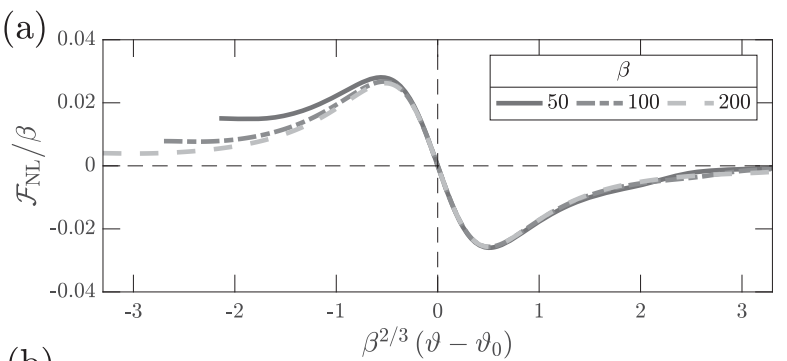

(b)

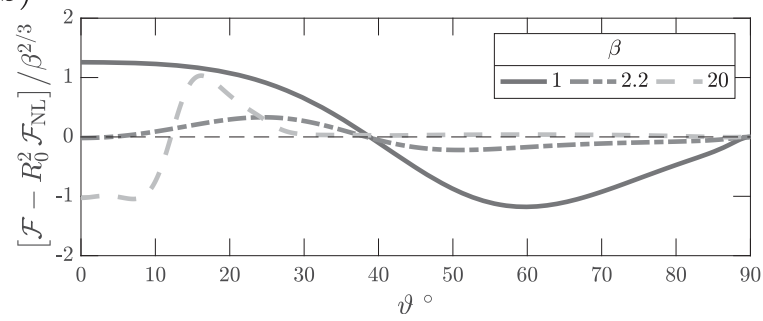

FIG. 9. (a) The contribution $\mathscr{F}_{\mathrm{NL}}$ to the coefficient $c_{3}$ from waves at angle $|\vartheta|$ in the limit of $\beta \gg 1$. $\mathscr{F}_{\mathrm{NL}}$ assumes a dipole pattern. The amplitude of each of the dipole peaks scale with $\beta$, and the widths of the dipole structure scale with $\beta^{-2 / 3}$. For $\beta \gg 1$ the structure of $\mathscr{F}_{\mathrm{NL}}$ is independent of the type of forcing used. (b) The contribution from waves at angle $|\vartheta|$ to the finite-amplitude equilibrium jet, as given by $\mathscr{F}-R_{0}^{2} \mathscr{F}_{\mathrm{NL}}$ for three values of $\beta$.

and is hindered by waves with angles $|\vartheta|>\vartheta_{0}$, while the equilibrated jet is supported through the upgradient fluxes of waves with angles $|\vartheta|>\vartheta_{0}$. The reason is that the amplitude $R_{0}(0)$ is so large that the sign of the integrand in (33) is reversed. Further investigation of the eddy-mean flow interactions leading to this peculiar feedback is out of the scope of the current work and will be reported in a future study.

Further insight into the equilibration dynamics is gained by noting that the coefficient $c_{3}$ can be written as the sum of two separate contributions:

$$
c_{3}=c_{3}^{\mathrm{ec}}+c_{3}^{1,2},
$$

which represent different physical processes (details on the decomposition can be found in appendix B). These contributions correspond to the two $O\left(\mu^{3}\right)$ possible interactions between the perturbed components of the mean flow $\mu \bar{u}_{1}$ and $\mu^{2} \bar{u}_{2}$ with the covariance corrections $\mu C_{1}, \mu^{2} C_{20}$, and $\mu^{2} C_{22}$.

Coefficient

$$
c_{3}^{\mathrm{ec}} \propto-f\left(0 \mid \bar{u}_{1}, C_{20}\right),
$$

is proportional to the mean vorticity flux feedback from the interaction of $\mu \bar{u}_{1}$ with the homogeneous covariance correction $\mu^{2} C_{20}$ to the equilibrium $C^{e}$. It measures the compensation in the vorticity flux as eddies lose energy to the mean flow.

\section{Coefficient}

$c_{3}^{1,2} \propto-f\left(0 \mid \bar{u}_{1}, C_{22} e^{2 i n_{c}\left(y_{a}+y_{b}\right) / 2}+\right.$ c.c. $)-f\left(0 \mid \bar{u}_{2}, C_{1}\right)$,

measures the mean vorticity flux feedbacks from the interaction of $\mu \bar{u}_{1}$ and $\mu^{2} \bar{u}_{2}$ with the inhomogeneous covariance corrections $\mu C_{1}$ and $\mu^{2} C_{22} e^{2 i n_{c}\left(y_{a}+y_{b}\right) / 2}$ to the equilibrium $C^{e}$. The exact form of the coefficients is given in (B16) and (B18), respectively.

Figure $8 \mathrm{~b}$ shows the contribution of the two processes in $c_{3}$ as a function of $\beta$. We observe that the main contribution to the coefficient $c_{3}$ comes from $c_{3}^{\text {ec }}$ for most values of $\beta$. Only for $\beta \ll 1$ is there a contribution from $c_{3}^{1,2}$ at the same order. ${ }^{3}$ The same results also hold for the case of the anisotropic forcing (see Fig. C1). Therefore, we conclude that, for most values of $\beta$, the mean flow is stabilized by the change in the homogeneous part of the covariance because of conservation of the total energy that leads to a concomitant reduction of the upgradient fluxes. For $\beta \ll 1$ there is no change in the eddy-mean flow dynamical processes involved, while for $\beta \gg 1$ the equilibrated flow is supported by the upgradient fluxes of the eddies that were initially hindering its formation.

\section{b. Equilibration processes for the upper-branch jets}

We have seen in the discussion surrounding Fig. 6 that the $2 n_{c}$ components of the upper-branch equilibria are much stronger than the corresponding $2 n_{c}$ components of the lower-branch jets. Therefore, we expect the interaction between the jet components with wavenumbers $n_{c}$ and $2 n_{c}$ to play an important role in the equilibration of the upper-branch jets. This is not at all the case for the lower-branch G-L equilibria for which this interaction quantified by $c_{3}^{1,2}$ is subdominant compared to the energy correction term $c_{3}^{\mathrm{ec}}$.

To investigate the interaction between the jet components with wavenumbers $n_{c}$ and $2 n_{c}$, we impose a mean flow with power only at those Fourier components:

$$
\bar{u}=\widehat{\bar{u}}_{1} e^{i n_{c} y}-\widehat{\bar{u}}_{2} e^{2 i n_{c} y}+\text { c.c. }
$$

At low supercriticality there is a phase difference of $180^{\circ}$ between the two components [see (22a) and the fact that $\left.\alpha_{2}<0\right]$. Therefore, we built in this phase difference in (37). We then compute the vorticity fluxes that are induced by the mean flow, (37), by employing the adiabatic approximation, that is, by assuming that the mean flow evolves slow enough that it remains in equilibrium with the eddy covariance, and thus, $\partial_{t} C \approx 0$. Such an

\footnotetext{
${ }^{3}$ Further analysis on the relative contributions of the forced eddies for the two distinct processes can be found in appendix B.
} 
adiabatic approximation is exact for the fixed points of the S3T dynamics, but it has also been proven adequate in qualitatively illuminating the eddy-mean flow dynamics away from the homogeneous or inhomogeneous equilibria (Farrell and Ioannou 2003, 2007; Bakas and Ioannou 2013b; Bakas et al. 2015). With the adiabatic approximation, the eddy covariance equation, (5b), simplifies to

$-\mathscr{L} C+\mathscr{N}\left(\widehat{\bar{u}}_{1} e^{i n_{c} y}-\widehat{\bar{u}}_{2} e^{2 i n_{c} y}+\right.$ c.c., $\left.C\right)+\varepsilon Q=0$.

We solve (38) for $C$, compute the vorticity fluxes, and decompose them into their Fourier components:

$$
\underbrace{\overline{v^{\prime} \zeta^{\prime}}}_{=\mathscr{R}(C)}=\sum_{m} \hat{f}_{m n_{c}}\left(\widehat{\bar{u}}_{1}, \widehat{\bar{u}}_{2}\right) e^{i m n_{c} y}+\text { c.c. }
$$

with $m$ positive integer. Then, from the mean flow equation, (5a), we obtain that the mean-flow components satisfy

$$
\begin{aligned}
& \frac{d \widehat{\bar{u}}_{1}}{d t}=\hat{f}_{n_{c}}\left(\widehat{\bar{u}}_{1}, \widehat{\bar{u}}_{2}\right)-\widehat{\bar{u}}_{1}, \\
& \frac{d \widehat{\bar{u}}_{2}}{d t}=\hat{f}_{2 n_{c}}\left(\widehat{\bar{u}}_{1}, \widehat{\bar{u}}_{2}\right)-\widehat{\bar{u}}_{2} .
\end{aligned}
$$

Figure 10 shows the mean-flow growth rates [e.g., $\left.\left(1 / \widehat{\bar{u}}_{1}\right) d \widehat{\bar{u}}_{1} / d t\right]$ as a function of the components $\widehat{\bar{u}}_{1}$ and $\widehat{\bar{u}}_{2}$ of the imposed mean flow. We see that for an infinitesimal mean flow (lower-left corner of the two panes; noted as region G-L), the growth of $\widehat{\bar{u}}_{1}$ resulting from the linear instability and the growth of $\widehat{\bar{u}}_{2}$ resulting from the second-order self-interaction of the unstable mode [cf. (22a)] lead to an increase of both $\widehat{\bar{u}}_{1}$ and $\widehat{\bar{u}}_{2}$. The flow, thus, equilibrates at the point of intersection of the zero contours for both mean-flow tendencies (thick black solid and dashed curves). This is the lower-branch $\mathrm{G}-\mathrm{L}$ equilibrium that is shown by the open circle and was discussed in the previous section.

There exist, however, two additional points of intersection, both of which are accessible to the flow through paths in the $\widehat{\bar{u}}_{1}-\widehat{\bar{u}}_{2}$ parameter space. If we start with a strong $\widehat{\bar{u}}_{1} \gtrsim 0.14$ component from point $\mathrm{A}$ in the figure, the large positive growth rate $\left(1 / \widehat{\bar{u}}_{2}\right) d \widehat{\bar{u}}_{2} / d t$ leads to a rapid increase of $\widehat{\bar{u}}_{2}$, while the slightly negative tendency $\left(1 \widehat{\bar{u}}_{1}\right) d \widehat{\bar{u}}_{1} / d t$ gradually weakens $\widehat{\widehat{u}}_{1}$ so that $\widehat{\bar{u}}_{2}$ and $\widehat{\bar{u}}_{1}$ move toward the right point of intersection. We perform an integration of the S3T dynamical system (5) with initial conditions starting from point $A$. The path that the dynamical system follows in the $\widehat{\bar{u}}_{1}-\widehat{\bar{u}}_{2}$ parameter space is shown by the dotted line and confirms the qualitative picture obtained via the mean-flow growth rates with the rapid increase of $\widehat{\bar{u}}_{2}$ and the eventual equilibration at the right point of intersection shown by the filled triangle. Similarly, if we start with a strong $\widehat{\bar{u}}_{2} \gtrsim 0.2$ component from point $\mathrm{B}$, the strong growth $\left(1 \widehat{\bar{u}}_{1}\right) d \widehat{\bar{u}}_{1} / d t$ and the weak negative tendency $\left(1 / \widehat{\bar{u}}_{2}\right) d \widehat{\bar{u}}_{2} / d t$ lead again to the equilibration of the flow through the path shown in Fig. 10. The growth rates close to the other point of intersection shown by the open circle reveals that this corresponds to an unstable equilibrium, and this is also confirmed through integrations of the S3T system, (5). These two points therefore correspond to the stable and unstable equilibria of the upper branch that are shown in Fig. 6.

The qualitative agreement between the approximate dynamics of the reduced dynamical system (40) and the nonlinear S3T dynamics reveal that it could be a useful tool for exploring the phase space of the S3T system. For example, the bifurcation structure of Fig. 6 could be obtained by plotting the growth rates obtained using the adiabatic approximation. Figure 11 shows the curves of zero tendencies for various values of the supercriticality. For low subcritical values $\varepsilon / \varepsilon_{c}<0.89$ (Fig. 11a), there is no point of intersection; therefore, only the homogeneous equilibrium exists. For $0.89 \leq \varepsilon / \varepsilon_{c}<1$ (Fig. 11b), there are two points of intersection revealing the existence of the stable and the unstable upper-branch equilibria, while for $1 \leq \varepsilon / \varepsilon_{c}$ (Fig. 11c), there is the additional lower-branch point. Finally, for highly supercritical values (Fig. 11d), there is only one point of intersection revealing the existence of the stable upper-branch equilibrium.

To shed light into the dynamics underlying these new equilibration paths that lead to the upper-branch equilibria, we decompose the eddy covariance as a Fourier sum over the inhomogeneous components:

$$
C=\sum_{m=0}^{4} \widehat{\bar{C}}_{m}\left(\mathbf{x}_{a}-\mathbf{x}_{b}\right) e^{i m n_{c}\left(y_{a}+y_{b}\right) / 2} .
$$

The sum is over five components. The reason is that the flux feedback on $\widehat{\bar{u}}_{1}$ and $\widehat{\bar{u}}_{2}$ is generated by the $n_{c}$ and $2 n_{c}$ components of the covariance. Inspection of the nonlinear term in (38) reveals that only the homogeneous component $\widehat{\bar{C}}_{0}$ as well as the covariance components at $n_{c}, 2 n_{c}, 3 n_{c}$, and $4 n_{c}$ can interact with the mean flow via (37) to yield these two covariance components. We then decompose the vorticity fluxes as

$$
\begin{aligned}
& \hat{f}_{n_{c}}\left(\widehat{\bar{u}}_{1}, \widehat{\bar{u}}_{2}\right)=f_{1,0}+f_{1,2}+f_{2,3}, \\
& \hat{f}_{2 n_{c}}\left(\widehat{\bar{u}}_{1}, \widehat{\bar{u}}_{2}\right)=f_{2,0}+f_{1,1}+f_{1,3}+f_{2,4} .
\end{aligned}
$$



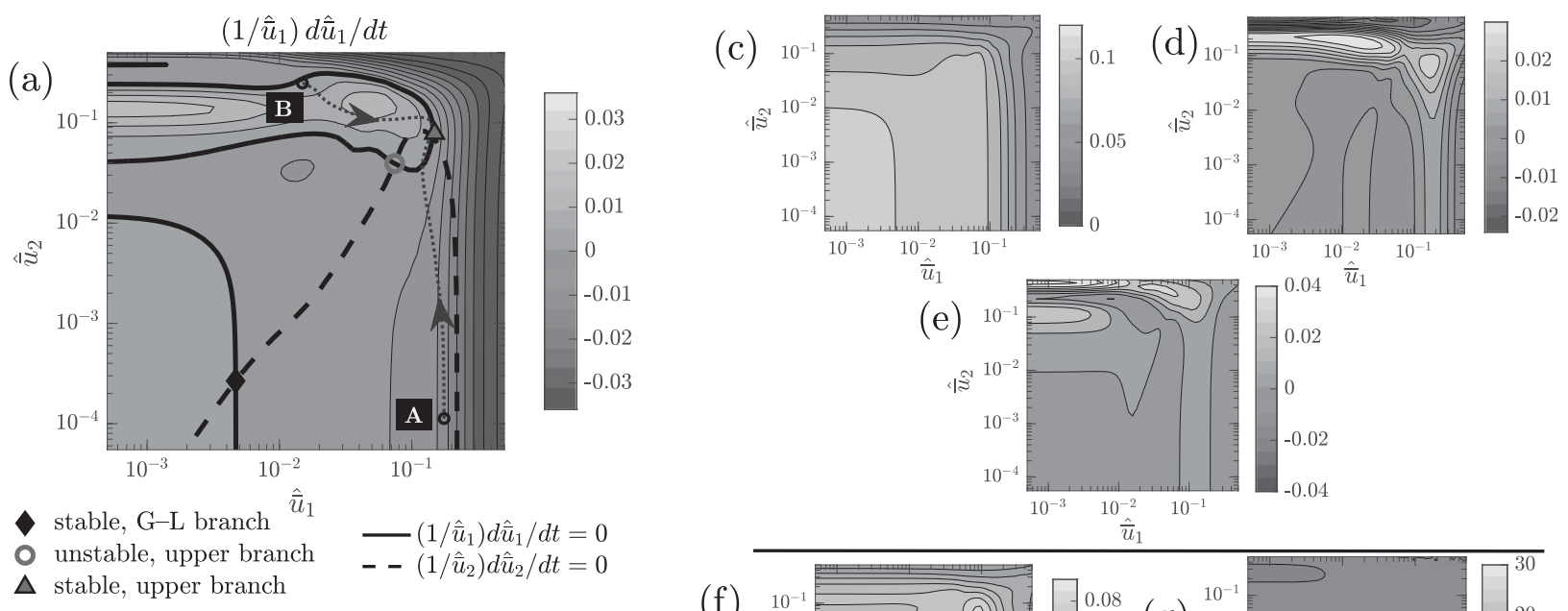
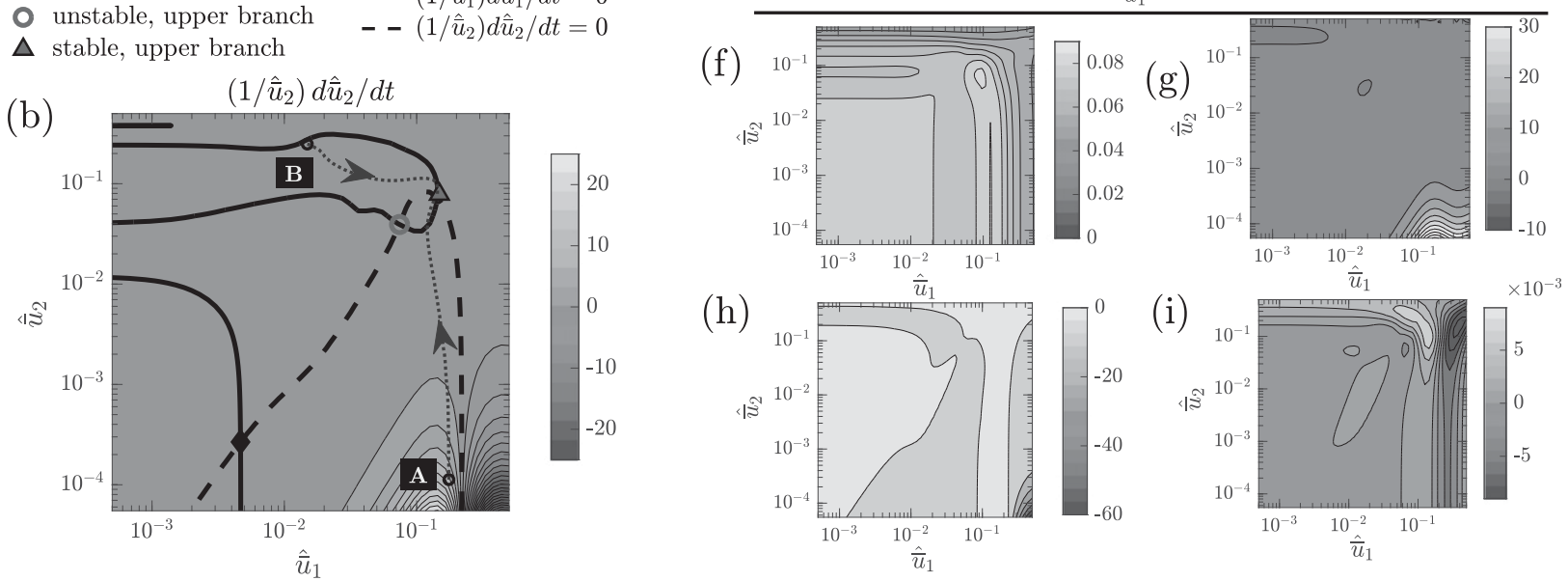

(f)

FIG. 10. (left) The mean-flow growth rates (a) $\left(1 / \widehat{\bar{u}}_{1}\right) d \widehat{\bar{u}}_{1} / d t$ and (b) $\left(1 / \widehat{\bar{u}}_{2}\right) d \widehat{\bar{u}}_{2} / d t$ obtained under an adiabatic approximation $\left(\partial_{t} C=0\right)$ for a mean flow $\bar{u}=\widehat{\bar{u}}_{1} e^{i n_{c} y}-\widehat{\bar{u}}_{2} e^{2 i n_{c} y}$ as a function of $\widehat{\bar{u}}_{1}$ and $\widehat{\bar{u}}_{2}$. The thick curves are the zero-tendency contours (solid curve for the $d \widehat{\bar{u}}_{1} / d t=0$ and dashed for $d \hat{\bar{u}}_{2} / d t=0$ ). Infinitesimal jet perturbations start in the region in the $\widehat{\bar{u}}_{1}-\widehat{\bar{u}}_{2}$ phase space denoted as G-L and end up in the lower-branch equilibrium shown by the filled circle. The arrows denote paths in the $\widehat{\bar{u}}_{1}-\widehat{\bar{u}}_{2}$ phase space that connect a finite-amplitude jet perturbation starting from points $\mathrm{A}$ and $\mathrm{B}$ and ending up to the upper-branch equilibrium, denoted by the filled triangle. [The paths were obtained by time stepping the S3T system, (5).] (top right) The breakdown of the flux feedback $\hat{f}_{n_{k} k_{*} *} / \widehat{\bar{u}}_{1}$ into the components (c) $f_{1,0} / \widehat{\bar{u}}_{1}$, (d) $f_{1,2} \widehat{\bar{u}}_{1}$, and (e) $f_{2,3} / \widehat{\bar{u}}_{1}$. (bottom right) The breakdown of $\hat{f}_{2 n_{c} k_{f} *} / \widehat{\bar{u}}_{2}$ into the components (f) $f_{2,0} / \bar{u}_{2}$, (g) $f_{1,1} / \widehat{\bar{u}}_{2}$, (h) $f_{1,3} / \widehat{\bar{u}}_{2}$, and (i) $f_{2,4} / \widehat{\bar{u}}_{2}$. Parameters used are $\beta_{58}$ and supercriticality $\mu=0.1$.

Each of the terms on the right-hand sides of (42) represents the different interactions among the mean-flow components $\widehat{\bar{u}}_{1}$ and $\widehat{\bar{u}}_{2}$ with the covariance components $\widehat{\bar{C}}_{0}, \widehat{\bar{C}}_{1}, \widehat{\bar{C}}_{2}, \widehat{\bar{C}}_{3}$, and $\widehat{\bar{C}}_{4}$. The first term in (42a) is proportional to the vorticity flux feedback from the interaction of $\widehat{\bar{u}}_{1}$ with the homogeneous covariance component $\bar{C}_{0}$ :

$$
f_{1,0} \propto f\left(0 \mid \widehat{\bar{u}}_{1} e^{i n_{c} y}, \widehat{\bar{C}}_{0}\right) .
$$

For low supercriticality,

$$
f_{1,0}-\widehat{\bar{u}}_{1} \approx A\left(1-c_{3}^{\mathrm{ec}}|A|^{2}\right) .
$$

This means that $f_{1,0}$ contains both the destabilizing feedback that drives the linear instability and the stabilizing feedback at finite amplitude that results from the energy correction. The terms $f_{1,2}$ and $f_{2,3}$ in (42a) describe the feedback of the nonlinear interaction between $\widehat{\bar{u}}_{1}$ and $\widehat{\widehat{u}}_{2}$ on $\widehat{\bar{u}}_{1}$ :

$$
\begin{aligned}
f_{1,2} \propto & f\left(0 \mid \widehat{\bar{u}}_{1}^{*} e^{-i n_{c} y}, \widehat{\bar{C}}_{2} e^{2 i n_{c}\left(y_{a}+y_{b}\right) / 2}\right) \\
& +f\left(0 \mid \widehat{\bar{u}}_{2} e^{2 i n_{c} y}, \widehat{\bar{C}}_{1}^{*} e^{-i n_{c}\left(y_{a}+y_{b}\right) / 2}\right), \\
f_{2,3} \propto & f\left(0 \mid \widehat{\bar{u}}_{2}^{*} e^{-2 i n_{c} y}, \widehat{\bar{C}}_{3} e^{3 i n_{c}\left(y_{a}+y_{b}\right) / 2}\right) .
\end{aligned}
$$

For low supercriticality,

$$
f_{1,2} \approx-c_{3}^{1,2} A|A|^{2},
$$

while $f_{2,3}$ is of higher order in $\mu$. Similarly, the second term on the right-hand side of (42b) is proportional to 

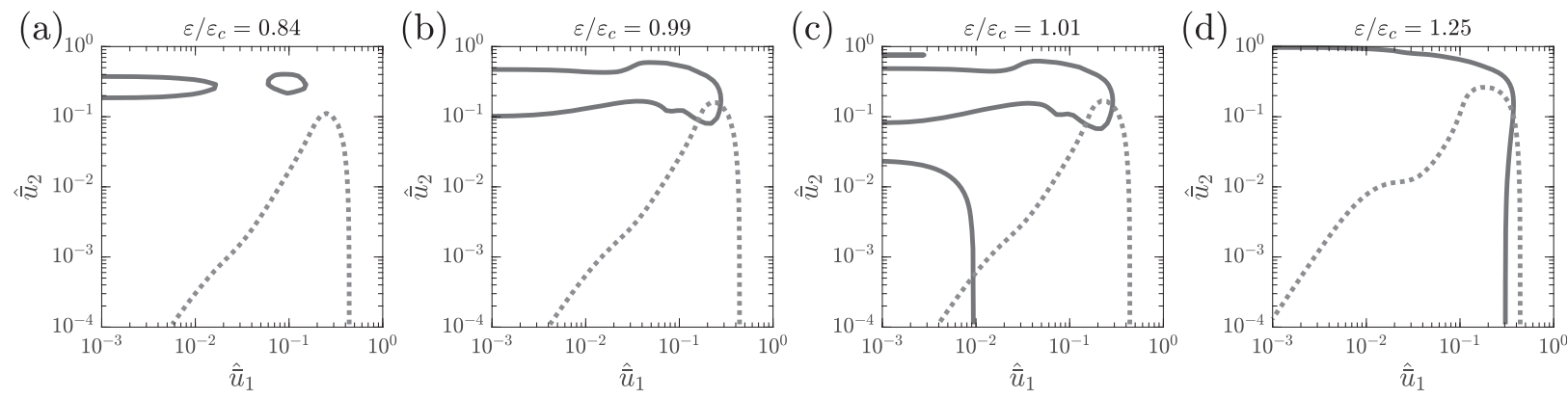

$-d \hat{\bar{u}}_{1} / d t=0 \cdots \cdots d \hat{\bar{u}}_{2} / d t=0$

FIG. 11. The locus of zero mean-flow tendencies in $\widehat{\bar{u}}_{1}-\widehat{\bar{u}}_{2}$ space for various supercriticalities for the case with $\beta_{58}$. Jet equilibria exist at the intersection of the two loci, when $d \widehat{\bar{u}}_{1} / d t=d \widehat{\bar{u}}_{2} / d t=0$. (a) $\varepsilon / \varepsilon_{c}=0.84$; there is no intersection as only the homogeneous equilibrium is stable for $\varepsilon / \varepsilon_{c}<0.89$ (see Fig. 6a). (b) $\varepsilon / \varepsilon_{c}=0.99$; there are two points of intersection that correspond to the stable and unstable upperbranch equilibria that exist for $0.89<\varepsilon / \varepsilon_{c}<1$. (c) $\varepsilon / \varepsilon_{c}=1.01$; there are three equilibria: two upper-branch equilibria (a stable and an unstable) and the lower-branch G-L equilibrium. (d) $\varepsilon / \varepsilon_{c}=1.25$; only one upper-branch equilibrium exists as the G-L branch and the unstable upper branch terminate at $\varepsilon / \varepsilon_{c}=1.068$.

the vorticity flux feedback from the interaction of $\widehat{\bar{u}}_{2}$ with the homogeneous covariance $\bar{C}_{0}$ :

$$
f_{2,0} \propto f\left(0 \mid \widehat{\bar{u}}_{2} e^{2 i n_{c} y}, \widehat{\bar{C}}_{0}\right)
$$

For low supercriticality, the flux feedback above is positive but does not overcome dissipation; that is, $0<f_{2,0}<\widehat{\bar{u}}_{2}$. Therefore, the homogeneous equilibrium is linearly stable with respect to jet perturbations with wavenumber $2 n_{c}$ (as expected). The terms $f_{1,1}, f_{1,3}$, and $f_{2,4}$ in (42b) describe the feedback of the nonlinear interaction between $\widehat{\bar{u}}_{1}$ and $\widehat{\bar{u}}_{2}$ on $\widehat{\bar{u}}_{2}$ :

$$
\begin{aligned}
& f_{1,1} \propto f\left(0 \mid \widehat{\bar{u}}_{1} e^{i n_{c} y}, \widehat{\bar{C}}_{1} e^{i n_{c}\left(y_{a}+y_{b}\right) / 2}\right), \\
& f_{1,3} \propto f\left(0 \mid \widehat{\bar{u}}_{1}^{*} e^{-i n_{c} y}, \widehat{\bar{C}}_{3} e^{3 i n_{c}\left(y_{a}+y_{b}\right) / 2}\right), \\
& f_{2,4} \propto f\left(0 \mid \widehat{\bar{u}}_{1}^{*} e^{-2 i n_{c} y}, \widehat{\bar{C}}_{4} e^{4 i n_{c}\left(y_{a}+y_{b}\right) / 2}\right) .
\end{aligned}
$$

For low supercriticality, $f_{1,1}$ drives the $\widehat{\bar{u}}_{2}$ component of the flow with an amplitude proportional to $\widehat{\bar{u}}_{1}^{2}$, and therefore, $\widehat{\bar{u}}_{2}$ equilibrates at amplitude $(22 \mathrm{a})$, while $f_{1,3}$ and $f_{2,4}$ are of higher order. Figures $10 \mathrm{c}-\mathrm{i}$ show the contribution of the various terms to the flux feedbacks $\hat{f}_{n_{c}}$ and $\hat{f}_{2 n_{c}}$, respectively. In the G-L region the fluxes are determined by $f_{0,1}, f_{0,2}$, and $f_{1,1}$. However, the "tongue" of positive tendency $\left(1 / \widehat{\bar{u}}_{1}\right) d \widehat{\bar{u}}_{1} / d t$ in Fig. 10a for large values of $\widehat{\bar{u}}_{2}$, as well as the region of very large positive tendency $\left(1 \widehat{\bar{u}}_{1}\right) d \widehat{\bar{u}}_{1} / d t$ in Fig. 10b, is determined by the other terms. As a result, the equilibration of the flow in the upper-layer branch is due to the nonlinear interaction of the two mean-flow components $\widehat{\bar{u}}_{1}$ and $\widehat{\bar{u}}_{2}$ rather than the energy correction that underlies the equilibration of the flow in the lower branch.

\section{Eckhaus instability of the side-band jets}

In this section we study the stability of the side-band jet equilibria. As noted by Parker and Krommes (2014), these harmonic jet equilibria are susceptible to Eckhaus instability, a well-known result for harmonic equilibria of the G-L equation (Hoyle 2006). Here, we present the main results of the Eckhaus instability and compare them with fully nonlinear S3T dynamics.

\section{a. An intuitive view of the Eckhaus instability}

To obtain intuition for the eddy-mean flow dynamics underlying the Eckhaus instability, note first that the G-L dynamics are given by the balance between the vorticity flux feedback $f_{r}(\nu)=f_{r}(0)-c_{2} \nu^{2}$, which provides a diffusive correction to the original upgradient fluxes $f_{r}(0)>0$ at $n_{c}$ and the stabilizing nonlinear term $c_{3}|A|^{2}$. Let us assume an equilibrium jet with $\nu>0$, that is, with a scale smaller than that of the most unstable jet at $n_{c}$, and also assume a sinusoidal phase perturbation:

$$
A(Y)=R_{0} e^{i[\nu Y+\eta \sin (q Y)]} \quad \text { with } \quad \eta \ll 1 .
$$

Figure 12 shows how the perturbed jet, (52), is compressed for half the wavelength of the phase perturbation $\pi / q$ (unshaded region) and dilated for the other half (shaded region). In the compressed region the jet appears with an enhanced wavenumber $\nu+\delta \nu$, while in the dilated region, the jet appears with a reduced wavenumber $\nu-\delta \nu$. As a result, the vorticity flux feedback $f_{r}(\nu)$ is larger in the dilated (shaded) region implying a tendency to enhance the jet; the opposite occurs in the compressed region (nonshaded). Figure 12 shows a qualitative sketch of the mean vorticity fluxes $\overline{v^{\prime} \zeta^{\prime}}$ that demonstrates this process. If the nonlinear 


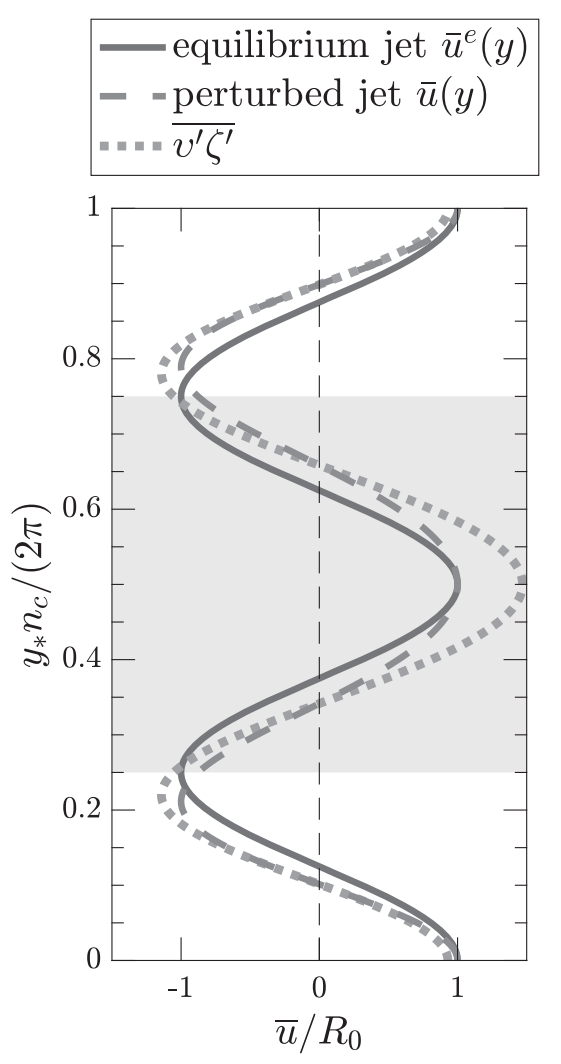

FIG. 12. Solid curve shows a sinusoidal equilibrium jet $\bar{u}^{e}=$ $R_{0} \cos \left[\left(n_{c}+\mu \nu\right) y\right]$ with smaller scale $\left(\nu=n_{c}\right)$ compared to the scale of the most unstable jet (we take $\mu=1$ so that the wavenumber differences with the most unstable jet are exaggerated for illustration purposes). Dashed curve shows the resulting jet when the phase of the equilibrium jet $\bar{u}^{e}$ is perturbed: $\bar{u}=$ $R_{0} \cos \left[\left(n_{c}+\mu \nu\right) y+\eta \sin (q y)\right]$, with $q=\nu$ and $\eta=1 / 2$ (for illustration purposes). This perturbation dilates the jet in the shaded region and compresses the jet in the unshaded region. Dash-dotted curve is a qualitative depiction of the expected vorticity flux feedback $\overline{v^{\prime} \zeta^{\prime}}$ for the perturbed jet based on the dependence of $f_{r}$ on the wavenumber $\nu$.

term does not counteract this mismatch, the dilated part of the jet will grow and take over the whole domain, thus producing a jet with lower $\nu$. (Similarly, for an equilibrium jet with $\nu<0$ there is a tendency for the compressed part of the jet to take over the whole domain producing a jet with larger $\nu$.)

To summarize, because of the diffusive nature of the vorticity flux feedback, there is a tendency to go toward $\nu=0$ jets if not counteracted by the nonlinear eddymean flow feedback.

\section{b. A formal view of the Eckhaus instability}

To address quantitatively the stability of the harmonic jet equilibria, (28), let us reformulate the G-L equation by rewriting the jet amplitude $A$ in polar form as

$$
A(Y, T)=R(Y, T) e^{i \Theta(Y, T)}
$$

where $R$ is the amplitude and $\Theta$ is the phase of the jet. The equilibrium jets have a constant amplitude $R_{0}(\nu)$ given by (28) and a linearly varying phase $\Theta=\nu Y$. From (19), such equilibria exist only for $|\nu|<\nu_{e}=\sqrt{1 / c_{2}}$. Consider now small perturbations about this equilibrium jet:

$$
R=R_{0}(\nu)+\hat{\rho} e^{i q Y+\lambda T}, \quad \Theta=\nu Y+\hat{\phi} e^{i q Y+\lambda T} .
$$

As shown in appendix $\mathrm{D}$, the perturbations grow exponentially if

$$
q^{2}+2\left(\nu_{e}^{2}-3 \nu^{2}\right)<0
$$

For an infinite domain, the gravest mode has $q=0$, and therefore, the jets with amplitude (53) are Eckhaus unstable when $|\nu|>\nu_{e} / \sqrt{3}$. Maximum instability occurs for

$$
|q|_{\max }=\nu_{e} \frac{\sqrt{3\left(\nu / \nu_{e}\right)^{4}+2\left(\nu / \nu_{e}\right)^{2}-1}}{2\left(\nu / \nu_{e}\right)},
$$

and therefore, the Eckhaus instability will form a jet of wavenumber $n_{c}+\mu\left(\nu \pm|q|_{\max }\right)$. Figure 13a shows the wavenumber $|q|_{\max }$ as a function of the equilibrium jet wavenumber $\nu$. Note that the equilibria with wavenumbers $\nu \approx \nu_{e} / \sqrt{3}$ are unstable to jets with neighboring wavenumbers as $|q|_{\max } \ll 1$, while equilibria with wavenumbers $\nu \approx \nu_{e}$ are unstable to the jet with wavenumber $n_{c}$ as $|q|_{\max } \approx 1$.

The growth rate for the most unstable structure with $|q|_{\max }$ is

$$
\lambda_{\max }=\frac{\left(3 \nu^{2}-\nu_{e}^{2}\right)^{2}}{4 c_{1} \nu_{e}^{2} \nu^{2}} .
$$

and is shown in Fig. 13b.

\section{c. Comparison with S3T dynamics}

We first compare the stability analysis for the harmonic jets derived in the weakly nonlinear limit of G-L dynamics to nonlinear dynamics in the S3T system. Note that the growth rate of the Eckhaus instability is much less than the corresponding growth rate of the flowforming instability of the homogeneous state of a jet for almost all wavenumbers $\nu$. Figure 13b compares the growth rate $\lambda_{\max }$ for the perturbation with $|q|_{\max }$ that will eventually form a jet with wavenumber $n_{c}+\mu\left(\nu \pm|q|_{\max }\right)$ to the growth rate of the flowforming instability of the homogeneous equilibrium that will form a jet with the same wavenumber (shown with dashed line). As a result, the weak Eckhaus instability manifests only in carefully contrived S3T 

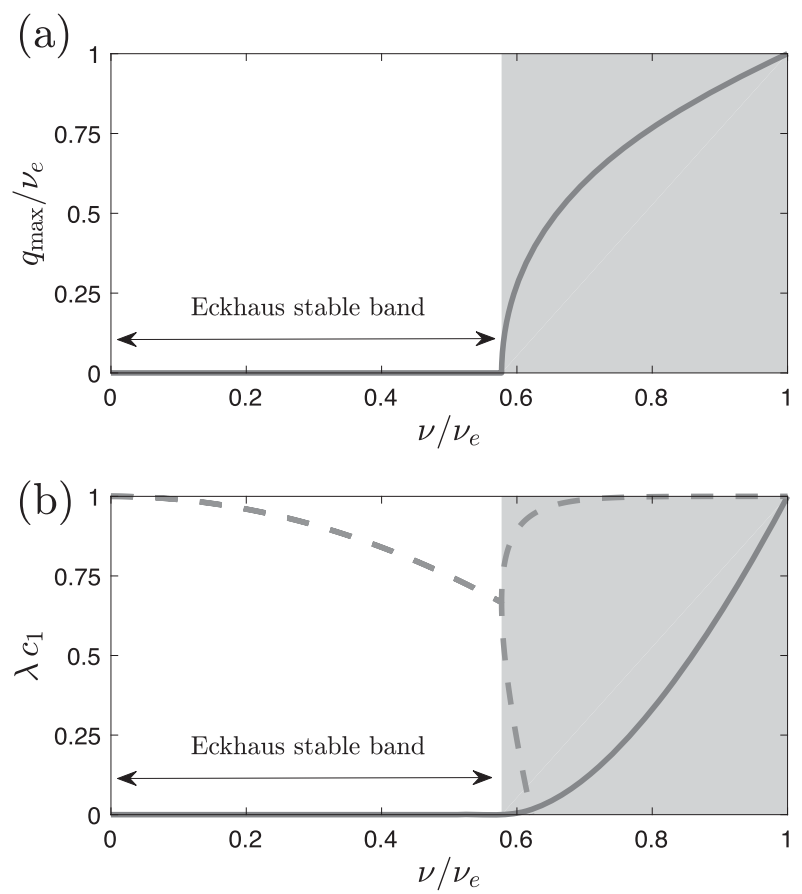

FIG. 13. (a) The most unstable wavenumber for the Eckhaus instability $|q|_{\max } / \nu_{e}$ as a function of the jet equilibrium wavenumber $\nu / \nu_{e}$. Instability occurs in the shaded region for $\nu / \nu_{e}>1 / \sqrt{3}$. (b) The growth rate for the most Eckhaus unstable jet with $q=q_{\max },(57)$, as a function of the jet equilibrium wavenumber $\nu$ (solid line). Also shown with a dashed line is the corresponding growth rate for the flow-forming instability of the jet with wavenumber $\nu \pm q_{\max }$ that will eventually be formed by the Eckhaus instability and is given by $\left[1-\left(\nu \pm q_{\max }\right)^{2} / \nu_{e}^{2}\right] / c_{1}$, according to the G-L equation, (27).

simulations; any simulation of the S3T system, (5), starting from a random initial perturbation at low supercriticality will evolve into the most unstable jet with wavenumber $n_{c}$.

Second, in contrast with the infinite domain, for the doubly periodic box, the first side-band jets appear when $\nu \geq \nu^{ \pm}$, while the gravest wavenumber $q$ is $q_{\min } \stackrel{\text { def }}{=}$ $1 /\left(\mu k_{f} L_{*}\right)$. Therefore, the instability criterion, (55), is satisfied for

$$
\mu \leq \frac{\sqrt{(3-1 / 2) c_{2}}}{k_{f *} L_{*}}
$$

We compare here the stability boundary, (58), with the stability analysis based on the nonlinear S3T dynamics. The stability of the inhomogeneous jet-turbulence S3T equilibria shown in Fig. 7 is studied using the numerical methods developed by Constantinou (2015) and Constantinou et al. (2016); for the stability boundary, (58), we use the effective values $c_{2}^{\mathrm{ex} \pm}$ for the sideband jet equilibria with $\nu^{ \pm}$. Unstable (stable) equilibria are shown in Fig. 7 with open (filled) symbols, while the stability boundaries for $\nu^{ \pm}$are shown with the vertical dotted lines. For $\beta_{6}$, the parabolic profile of the eigenvalue relation, on which the Eckhaus instability calculations are based, remains accurate for larger supercriticalities, and therefore, the stability boundary, (58), consists of a good approximation. For larger and smaller values of $\beta$, the parabolic profile is not so accurate, and therefore, the criterion developed fails. For example, for both $\beta_{1}$ and $\beta_{192}$, all the $\nu^{+}$jet equilibria are unstable.

Last, we compare the development of the Eckhaus instability as predicted by the G-L dynamics (27) and as predicted by the S3T dynamics. Figure 14 shows the evolution of the slightly perturbed $n_{*}=5\left(\nu^{-}\right)$and $n_{*}=$ $7\left(\nu^{+}\right)$equilibria for $\beta_{6}$ and supercriticality $\mu=0.3$ obtained from integrations of the S3T system (5). In both cases, the equilibria are unstable to $q=q_{\min }$ perturbations. As the instability develops the $\widehat{\bar{u}}\left(k_{y *}=6\right)$ component of the flow grows exponentially (Figs. 14c,d), and the flow moves into the stable $n_{*}=6\left(n_{c}\right)$ equilibrium jet by branching or merging (Figs. 14a,b). We compute the growth rate of the Eckhaus instability from (D4) by substituting $\nu=q=1 /\left(\mu k_{f} L_{*}\right)$ and using the effective values $c_{2}^{\text {ex }}$ :

$\lambda^{ \pm}=\mu^{2} \frac{-1+\sqrt{\left[\left(\mu^{\mathrm{ex} \pm} / \mu\right)^{2}-1\right]^{2}+4\left(\mu^{\mathrm{ex} \pm} / \mu\right)^{4}}}{c_{1}}$.

Figures $14 \mathrm{c}$ and $14 \mathrm{~d}$ demonstrate that the growth rate obtained by (59) is in excellent agreement with the growth rate of the Eckhaus instability in the nonlinear simulations. Furthermore, the equilibrium jet amplitude is accurately predicted by (29).

Figure 15 shows the comparison of the growth rates for the other unstable side-band jet equilibria illustrated in Fig. 7. We see once more that for $\beta_{6}$, for which the parabolic approximation of the eigenvalue relation used to obtain the $\mathrm{G}-\mathrm{L}$ dynamics is accurate, the growth rates agree for almost all the unstable range. For $\beta_{1}$ and $\beta_{192}$, for which the parabolic profile is not accurate, there is in general disagreement.

\section{Conclusions}

We examined the dynamics that underlies the formation and support of zonal jets at finite amplitude in forced-dissipative barotropic beta-plane turbulence using the statistical state dynamics of the turbulent flow closed at second order. Within this framework, jet formation is shown to arise as a flow-forming instability (or zonostrophic instability) of the homogeneous statistical equilibrium turbulent state when the 

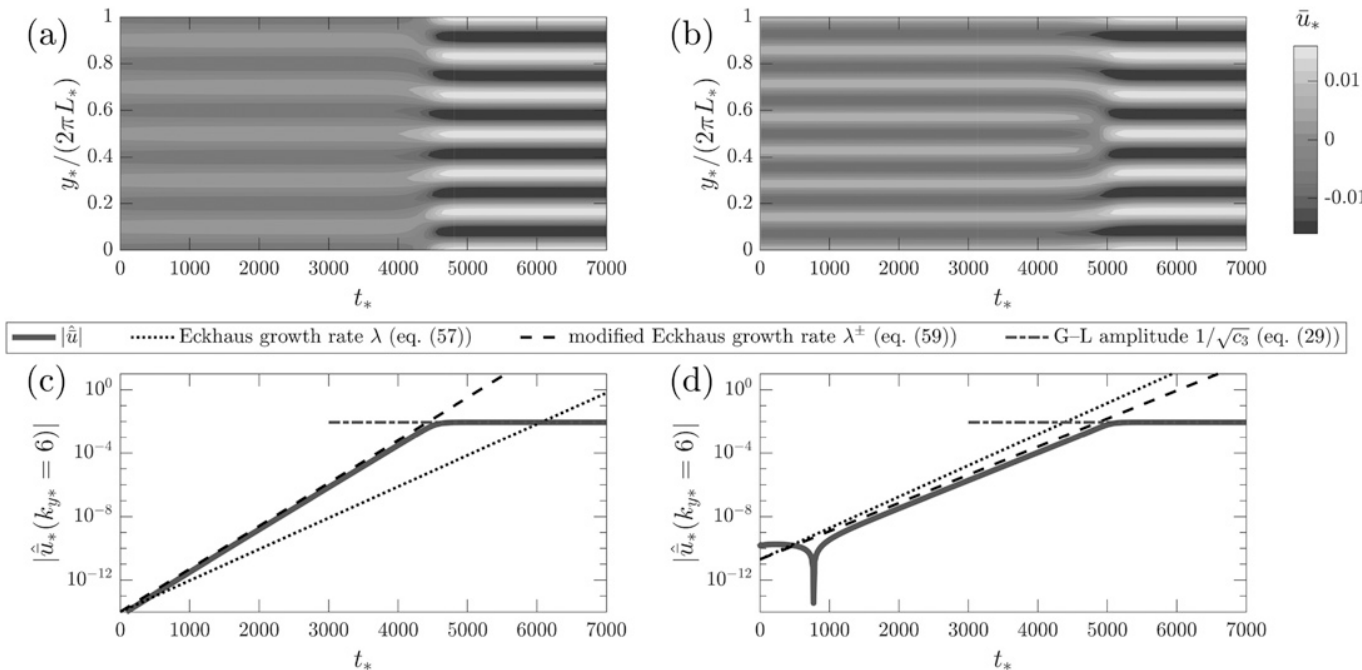

FIG. 14. The equilibration of the Eckhaus instability under S3T dynamics. (a) The evolution of the mean flow $\bar{u}_{*}(y, t)$ for the slightly perturbed $n_{*}=5\left(\nu^{-}\right)$equilibrium. (c) The evolution of the $n_{*}=6$ Fourier component of the flow (solid). (b) The growth rate predicted by (59) (dashed) and the amplitude of the $n_{*}=6$ jet as predicted by (29) (dash-dotted). (b),(d) As in (a) and (c), respectively, but for the slightly perturbed $n_{*}=7\left(\nu^{+}\right)$equilibrium. The planetary vorticity gradient is $\beta_{6}$, and the supercriticality is $\mu=0.3$.

nondimensional parameter $\varepsilon=\varepsilon_{*} /\left(k_{f^{*}}^{-2} r_{*}^{3}\right)$ crosses a certain critical threshold $\varepsilon_{c}$. In this work, we studied the dynamics that govern the equilibration of the flowforming instability in the limit of small supercriticality $\mu=\sqrt{\varepsilon / \varepsilon_{c}-1}$.

When supercriticality $\mu \ll 1$, the growth rate of the unstable modes as a function of the mean-flow wavenumber is to a good approximation a parabola. This allows a two-time, two-scale approximation of the nonlinear dynamics resulting in the weakly nonlinear Ginzburg-Landau dynamics for the evolution of zonal jets. The equilibration of the flow-forming instability was extensively investigated using the $\mathrm{G}-\mathrm{L}$ dynamics. Also, the predictions of the weakly nonlinear G-L dynamics regarding (i) the amplitude of the equilibrated jets and (ii) their stability were compared to the fully nonlinear S3T dynamics for a wide range of values for the nondimensional parameter $\beta=\beta_{*} /\left(k_{f} * r_{*}\right)$.

According to $\mathrm{G}-\mathrm{L}$ dynamics, the harmonic unstable modes of the homogeneous equilibrium state equilibrate at finite amplitude. The predicted amplitude of the jet that results from the equilibration of the most unstable mode with wavenumber $n_{c}$ was compared to the amplitude of the jet equilibria of the nonlinear S3T dynamics. For $\beta \lesssim 20$, the jet amplitude was found to be accurately predicted by the G-L dynamics for up to $\mu \approx 0.2$. For $\beta \gtrsim 20$, a new branch of jets with much larger amplitudes was discovered that was distinctly
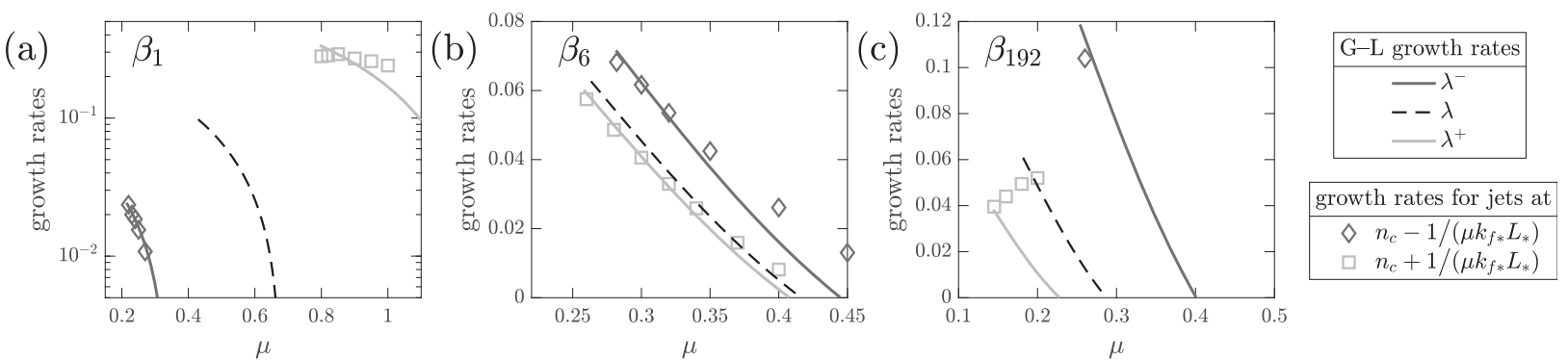

FIG. 15. Growth rate for the Eckhaus instability of the finite-amplitude jets. Shown is the growth rate as a function of supercriticality $\mu$ for (a) $\beta_{1}$, (b) $\beta_{6}$, and (c) $\beta_{192}$, obtained from the stability analysis for the equilibrium jets with wavenumbers $n_{c}-1 /\left(\mu k_{f} * L_{*}\right)($ diamonds) and $n_{c}+1 /\left(\mu k_{f *} L_{*}\right)$ (squares) using the fully nonlinear system, (5). Dashed curves show the growth rate as predicted from the $\mathrm{G}-\mathrm{L}$ dynamics; solid curves show the growth rate, (59), as predicted from the G-L dynamics using the modified values for $c_{2}$, while dashed curves show the unmodified growth rate, (D4). 
different from the G-L branch of jet equilibria. The bifurcation diagram (e.g., Fig. 6) exhibits a classic cusp bifurcation with hysteretic loops. The new branch of jet equilibria exists even at subcritical values of the flow-forming instability of the homogeneous state (i.e., for $\varepsilon<\varepsilon_{c}$ ). This has two consequences: First, continuation methods for finding equilibria converge only for small supercriticalities, as the jet equilibria transition discontinuously to the upper branch (see, e.g., Fig. 6a). This explains the failure to converge to equilibria reported by Parker and Krommes (2014). Second, the cusp bifurcation allows the emergence of jets at subcritical parameter values through a nonlinear flow-forming instability.

The amplitudes of the jets that emerge from the sideband jet instabilities of the most unstable mode of the flow-forming instability (i.e., the jets that emerge at scales $\left.n_{c^{*}} \pm 1 / L_{*}\right)$ were also compared. The amplitude predicted by the G-L equation is partially based on the parabolic approximation to the dispersion relation and, more specifically, on the curvature of the function of the growth rate at criticality. This approximation was found to be valid away from criticality only for nondimensional $\beta \approx 5$, and as a result, the predicted amplitude fails outside this range. We proposed a way to remedy this discrepancy (at least to some extend) by using the exact values for the curvature of the growth rate function for larger supercriticalities instead of the curvature given by the parabolic approximation (see, e.g., Fig. 14). With this modification, the side-band jet amplitudes can be predicted by the G-L dynamics close to their onset for $\beta \lesssim 1$ and for a wide range of supercriticalities for $\beta \approx 5$. For $\beta \gtrsim 20$, apart from the $\mathrm{G}-\mathrm{L}$ branch, the additional branch of higher-amplitude sideband jets was also found.

The physical and dynamical processes underlying the equilibration of the flow-forming instability were then examined using three methods. The first was the decomposition of the nonlinear term in the G-L equation governing the equilibration of the instability in two terms. One involves the change in the homogeneous part of the eddy covariance that is required by total energy conservation. The other involves the vorticity flux feedback resulting from the interaction of the most unstable jet with wavenumber $n_{c}$ and the jet with the double harmonic $2 n_{c}$ that is inevitably generated by the nonlinear interactions. The second was the method of Bakas et al. (2015) for separating the contributions of the various eddies in the induced vorticity fluxes: both for the linear term in the G-L equation that drives the instability and also for the nonlinear term that stabilizes the flow. In this way, the eddies yielding upgradient fluxes and the eddies yielding downgradient fluxes were identified along with the change in the upgradient or downgradient character of the fluxes that occurs as the jets grow. The third method was the development of a reduced dynamical system that retains the fully nonlinear interactions in contrast to the G-L equation. This reduced system is based on an adiabatic assumption for the covariance changes and on a Galerkin truncation of the dynamics retaining only the $n_{c}$ and $2 n_{c}$ components of the mean flow that play important roles in the equilibration of the zonostrophic instability.

For the G-L branch, the central physical process responsible for the equilibration is the reduction in the upgradient vorticity flux that occurs through the change in the homogeneous part of the eddy covariance. For low values of $\beta$, the instability is quickly quenched, and the jets equilibrate at low amplitude. The reason is that the contribution of the eddies that induce upgradient fluxes and drive the instability is weakened as the jets emerge, while simultaneously, the contribution of the eddies that induce downgradient fluxes is increased. As a result, the jets equilibrate at a small amplitude and are supported by the same eddies that drive the instability.

For large values of $\beta$, both the upgradient and the downgradient contributions are almost equally weakened, thus leading to a slow decay of the growth rate and to an equilibrated jet with a much larger amplitude. Because the equilibrium amplitude is large, the stabilizing fluxes that are multiplied by the square of the jet amplitude in the G-L equation are dominant, and therefore, at equilibrium, the jet is supported by the eddies that were initially hindering its growth (these eddies have phase lines that form small angles with the meridional but different than zero).

For the new branch of jet equilibria the main physical process responsible for the equilibration is the interaction of the $n_{c}$ and $2 n_{c}$ components of the emerging flow. Starting from a finite-amplitude jet with either strong $n_{c}$ or $2 n_{c}$ components, this nonlinear interaction leads to rapid growth of the jet and to equilibration of the flow at amplitudes much larger than the $\mathrm{G}-\mathrm{L}$ branch and with a much stronger $2 n_{c}$ component.

Finally, the stability of the equilibrated side-band unstable jet perturbations was examined. For an infinite domain, zonal jets with scales close to the scale $n_{c}$ of the most unstable mode of the flow-forming instability are stable; jets with scales much larger or much smaller are unstable. The incipient Eckhaus instability of the harmonic equilibria of the G-L equation is well studied within the literature of pattern formation, but here, it was interpreted in a physically intuitive way. The equilibrated jets have a low amplitude (proportional to the supercriticality) and therefore do not significantly 
change the structure of the turbulence. As a result, a mean-flow perturbation on the turbulent flow induces approximately the same vorticity flux feedback as in the absence of any jet with the vorticity flux feedback having a maximum at the most unstable wavenumber. Therefore, when a dilation-compression phase perturbation is inserted in the equilibrated jet that has a different wavenumber than $n_{c}$, the vorticity flux feedback for the dilated or the compressed part of the jet will be larger, and this part of the jet tends to grow and take over the whole domain.

The predictions for the stability boundary and the growth rate of the Eckhaus instability were then compared to the stability analysis of the jet equilibria using the fully nonlinear S3T system and the methods developed in Constantinou (2015). For $\beta \approx 5$, using the exact values for the curvature of the growth rate function yields accurate predictions for both the stability boundary and the growth rate. As the instability develops the unstable side-band jets with smaller (larger) scale than the jet with wavenumber $n_{c}$ branch (merge) into the stable $n_{c}$ jet. For low or high values of $\beta$, large quantitative discrepancies occur with a few exceptions, but the qualitative picture of the dynamics with branching (merging) into the stable jet equilibrium remains.

We note that the comparison of the G-L dynamics with nonlinear S3T integrations, as well as investigation of the equilibration process with an anisotropic ring forcing, showed that the results in this study are not sensitive to the forcing structure.

A question that rises naturally is whether the results discussed here are relevant for strong turbulent jets. Strong turbulent jets also undergo bifurcations as the turbulence intensity increases. There are, however, qualitative differences compared to weak jets: strong jets always merge to larger scales, while weak jets can either merge or branch to reach a scale close to $n_{c}$. Based on the relevant dynamics in pattern formation, we expect that the antidiffusive phase dynamics that are involved in the Eckhaus instability will play a significant role in the secondary instabilities of large-amplitude jets as well. Moreover, the generalization of the GinzburgLandau dynamics that we have put forward in this study [i.e., (40)] is able to describe the slow evolution of a jet that consists of more than just one harmonic. This generalization of the Ginzburg-Landau dynamics, we hope, will provide a vehicle for understanding the dynamics involving bifurcations of strong turbulent jets.

Acknowledgments. The authors thank Jeffrey B. Parker for helpful comments on the first version of the manuscript. N.A.B. was supported by the AXA Research
Fund. N.C.C. was partially supported by the NOAA Climate and Global Change Postdoctoral Fellowship Program, administered by UCAR's Cooperative Programs for the Advancement of Earth System Sciences and also by the National Science Foundation under Award OCE-1357047.

\section{APPENDIX A}

\section{S3T Formulation and Eigenvalue Relation of the Flow-Forming Instability}

In this appendix we derive the eigenvalue relation of the flow-forming instability. The eigenvalue relation was first derived by Srinivasan and Young (2012). Here, we repeat the derivation mainly to introduce some notation and terminology that will prove to be helpful in understanding the nonlinear equilibration of the flowforming instability.

Consider the S3T system, (5), where

$$
\mathscr{C} \stackrel{\text { def }}{=} \beta\left(\partial_{x_{a}} \Delta_{a}^{-1}+\partial_{x_{b}} \Delta_{b}^{-1}\right)+2
$$

is the operator governing the linear eddy dynamics,

$$
\begin{aligned}
\mathscr{N}(\bar{u}, C) \stackrel{\text { def }}{=} & {\left[-\bar{u}_{a} \partial_{x_{a}}+\left(\partial_{y_{a}}^{2} \bar{u}_{a}\right) \partial_{x_{a}} \Delta_{a}^{-1}\right.} \\
& \left.-\bar{u}_{b} \partial_{x_{b}}+\left(\partial_{y_{b}}^{2} \bar{u}_{b}\right) \partial_{x_{b}} \Delta_{b}^{-1}\right] C,
\end{aligned}
$$

is the nonlinear operator governing the eddy-mean flow interaction, and

$$
\mathscr{R}(C) \stackrel{\text { def }}{=} \frac{1}{2}\left[\left(\partial_{x_{a}} \Delta_{a}^{-1}+\partial_{x_{b}} \Delta_{b}^{-1}\right) C\right]_{a=b},
$$

is the eddy vorticity flux driving the mean flow.

The eigenvalue relation is obtained by linearizing the S3T system, (5), about the homogeneous equilibrium, (8). Then, introducing the ansatz (9) in the linearized S3T equations, we obtain

$$
\begin{gathered}
(\sigma+1) \delta \bar{u}=\mathscr{R}(\delta C), \\
(\sigma+\mathscr{L}) \delta C=\mathscr{N}\left(\delta \bar{u}, C^{e}\right) .
\end{gathered}
$$

The quantity

$$
f(\sigma \mid \delta \bar{u}, C) \stackrel{\text { def }}{=} \mathscr{B}\left[(\sigma+\mathscr{L})^{-1} \mathscr{N}(\delta \bar{u}, C)\right]
$$

is the vorticity flux induced by the distortion of the incoherent homogeneous eddy equilibrium field with covariance $C$ by the mean flow $\delta \bar{u}$.

The inversion of the operators and the algebra are simplified by taking the Fourier decomposition of $\tilde{C}_{n}^{(h)}$ : 


$$
\tilde{C}_{n}^{(h)}\left(\mathbf{x}_{a}-\mathbf{x}_{b}\right)=\int \frac{d^{2} \mathbf{k}}{(2 \pi)^{2}} \hat{C}(\mathbf{k}) e^{i \mathbf{k} \cdot\left(\mathbf{x}_{a}-\mathbf{x}_{b}\right)} .
$$

By inserting (A6) and (8) into (A4b), we obtain

$$
\delta C=\varepsilon e^{i n\left(y_{a}+y_{b}\right) / 2}\left[G^{+}\left(\sigma \mid \mathbf{x}_{a}-\mathbf{x}_{b}\right)-G^{-}\left(\sigma \mid \mathbf{x}_{a}-\mathbf{x}_{b}\right)\right],
$$

where we defined

$$
G^{ \pm}(\sigma \mid \mathbf{x}) \stackrel{\text { def }}{=} \int \frac{d^{2} \mathbf{k}}{(2 \pi)^{2}} \frac{i k_{x} k_{\mp}^{2}\left(k_{ \pm}^{2}-n^{2}\right)}{(\sigma+2) k_{+}^{2} k_{-}^{2}+2 i \beta n k_{x} k_{y}} \frac{\hat{Q}\left(\mathbf{k}_{ \pm}\right)}{2} e^{i \mathbf{k} \cdot \mathbf{x}},
$$

with $\mathbf{k}_{ \pm}=\mathbf{k}+\mathbf{n} / 2, \mathbf{n}=(0, n)$, and $k_{ \pm}=\left|\mathbf{k}_{ \pm}\right|$. Inserting (A7) in (A4a), we obtain (10), in which

$f=\int \frac{d^{2} \mathbf{k}}{(2 \pi)^{2}} \frac{n k_{x}^{2}\left(k_{y}+n / 2\right)\left(1-n^{2} / k^{2}\right)}{(\sigma+2) k^{2} k_{s}^{2}+2 i \beta n k_{x}\left(k_{y}+n / 2\right)} \hat{Q}(\mathbf{k})$,

with $k_{s} \stackrel{\text { def }}{=}|\mathbf{k}+\mathbf{n}|$. After substituting the ring forcing power spectrum, (4), expressing the integrand in polar coordinates $\left(k_{x}, k_{y}\right)=(k \cos \vartheta, k \sin \vartheta)$, and integrating over $k$, (A9) becomes

$$
f=\int_{0}^{2 \pi} \frac{N_{f} d \theta}{(\sigma+2) D_{f}+i \beta D_{\beta}},
$$

with $N_{f}(\theta)=n \cos ^{2} \theta(\sin \vartheta+n / 2)\left(1-n^{2}\right) / \pi, \quad D_{f}(\vartheta)=$ $\cos ^{2} \vartheta+(\sin \vartheta+n)^{2}$, and $D_{\beta}(\vartheta)=2 n \cos \vartheta(\sin \vartheta+n / 2)$. At criticality $(\sigma=0)$, using the mirror symmetry property of the forcing, that is, $\hat{Q}\left(-k_{x}, k_{y}\right)=\hat{Q}\left(k_{x}, k_{y}\right)$, the vorticity flux feedback is rewritten as

$$
f_{r}=\int_{0}^{\pi / 2} \mathscr{F}(\vartheta, n) d \vartheta
$$

where

$$
\begin{aligned}
\mathscr{F}(\vartheta, n)= & \frac{N_{f}(\vartheta) D_{f}(\vartheta)}{4 D_{f}^{2}(\vartheta)+\beta^{2} D_{\beta}^{2}(\vartheta)} \\
& +\frac{N_{f}(\vartheta+\pi) D_{f}(\vartheta+\pi)}{4 D_{f}^{2}(\vartheta+\pi)+\beta^{2} D_{\beta}^{2}(\vartheta+\pi)}
\end{aligned}
$$

is the contribution to the feedback from the waves with wavevectors $\left(k_{x}, k_{y}\right)$ and $\left(-k_{x},-k_{y}\right)$, and their mirror symmetric wavevectors $\left(-k_{x}, k_{y}\right)$ and $\left(k_{x},-k_{y}\right)$, respectively.

\section{APPENDIX B}

\section{Ginzburg-Landau Equation for the Weakly Nonlinear Evolution of a Zonal Jet Perturbation about the Homogeneous State}

To obtain the G-L equation governing the nonlinear S3T dynamics near the onset of the instability, we assume that the energy input rate is slightly supercritical $\varepsilon=\varepsilon_{c}\left(1+\mu^{2}\right)$, where $\mu \ll 1$ measures the supercriticality. As discussed in section 4 , the emerging jet grows slowly at a rate $O\left(\mu^{2}\right)$ and contains a band of wavenumbers of $O(\mu)$ around $n_{c}$, where $n_{c}$ is the wavenumber of the jet that achieves neutrality at $\varepsilon_{c}$. Therefore, we assume that the dynamics evolve on a slow time scale $T=\mu^{2} t$ and are modulated at a long meridional scale $Y=\mu y$. The leading-order jet is $\bar{u}_{1}=$ $A(Y, T) e^{i n_{c} y}$. We then expand the velocity and the covariance as a series in $\mu$ :

$$
\begin{aligned}
\bar{u}= & \mu \bar{u}_{1}(y, Y, T)+\mu^{2} \bar{u}_{2}(y, Y, T)+O\left(\mu^{3}\right), \\
C= & C^{e}\left(\mathbf{x}_{a}-\mathbf{x}_{b}\right)+\mu C_{1}\left(\mathbf{x}_{a}-\mathbf{x}_{b}, Y_{a}, Y_{b}, T\right) \\
& +\mu^{2} C_{2}\left(\mathbf{x}_{a}-\mathbf{x}_{b}, Y_{a}, Y_{b}, T\right)+O\left(\mu^{3}\right),
\end{aligned}
$$

along with the linear and nonlinear operators $\mathscr{B}$ and $\mathscr{N}$ that depend on the fast and slow meridional coordinates, $y$ and $Y$, respectively.

We substitute (B1) in (5) and collect terms with equal powers of $\mu$. As discussed in section 4 , we further assume that the amplitude $A$, as well as $C_{1}$ and $C_{2}$, are independent of the slow coordinate $Y$. This way, operators $\mathscr{L}$ and $\mathscr{N}$ also become independent of $Y$. In this case, the order- $\mu^{0}$ terms yield the homogeneous equilibrium. Terms of order $\mu^{1}$ yield the balance

$$
\mathscr{C}\left(\begin{array}{c}
\bar{u}_{1} \\
C_{1}
\end{array}\right) \stackrel{\text { def }}{=}\left(\begin{array}{c}
\bar{u}_{1}-\mathscr{R}\left(C_{1}\right) \\
\mathscr{L} C_{1}-\mathscr{N}\left(\bar{u}_{1}, C^{e}\right)
\end{array}\right)=0
$$

which can also be compactly written as

$$
\bar{u}_{1}=\varepsilon_{c} f\left(0 \mid \bar{u}_{1}, Q / 2\right),
$$

where $f\left(\sigma \mid \bar{u}_{1}, Q / 2\right)$ is the vorticity flux feedback on the mean flow $\bar{u}_{1}$ as defined in (A5). The solution of (B2) is the eigenfunction of operator $\mathscr{t}$ with zero eigenvalue:

$$
\left(\begin{array}{l}
\bar{u}_{1} \\
C_{1}
\end{array}\right)=A(T)\left(\begin{array}{c}
e^{i n_{c} y} \\
\varepsilon_{c} \mathrm{e}^{i n_{c}\left(y_{a}+y_{b}\right) / 2}\left[G_{c}^{+}\left(0 \mid \mathbf{x}_{a}-\mathbf{x}_{b}\right)-G_{c}^{-}\left(0 \mid \mathbf{x}_{a}-\mathbf{x}_{b}\right)\right]
\end{array}\right)+\text { c.c. }
$$


In (B4) the subscript $c$ on terms $G^{ \pm}$denotes that they are evaluated at $n=n_{c}$. At order $\mu^{2}$ the balance is

$$
\mathscr{b}\left(\begin{array}{l}
\bar{u}_{2} \\
C_{2}
\end{array}\right)=\left(\begin{array}{c}
0 \\
\mathscr{N}\left(\bar{u}_{1}, C_{1}\right)+\varepsilon_{c} Q
\end{array}\right) .
$$

Equation (B5) has a homogeneous solution that is proportional to $\left[\bar{u}_{1}, C_{1}\right]^{\mathrm{T}}$ and can be incorporated in it, and a particular solution. The nonlinear term $\mathscr{N}\left(\bar{u}_{1}, C_{1}\right)$ generates both a double- and a zeroharmonic mean flow (and covariance). As a result, the particular solution is

$$
\begin{aligned}
\left(\begin{array}{c}
\bar{u}_{2} \\
C_{2}
\end{array}\right)= & \left(\begin{array}{c}
0 \\
\varepsilon_{c} Q\left(\mathbf{x}_{a}-\mathbf{x}_{b}\right) / 2+C_{20}\left(\mathbf{x}_{a}-\mathbf{x}_{b}, T\right)
\end{array}\right) \\
& +\left(\begin{array}{c}
\alpha_{2} A(T)^{2} e^{2 i n_{c} y} \\
C_{22}\left(\mathbf{x}_{a}-\mathbf{x}_{b}, T\right) e^{2 i n_{c}\left(y_{a}+y_{b}\right) / 2}
\end{array}\right)+\text { c.c. }
\end{aligned}
$$

where $C_{20}$ and $C_{22}$ are the zero- and double-harmonic coefficients of the covariance and

$$
\alpha_{2} \stackrel{\frac{\varepsilon_{c}}{2} \int \frac{d^{2} \mathbf{k}}{(2 \pi)^{2}} \frac{i n_{c} k_{x}^{3}\left(k^{2}-n_{c}^{2}\right)}{k^{2} k_{2}^{2}+i \beta n_{c} k_{x} k_{y, 1}}\left[\frac{k_{y, 2}\left(k_{2}^{2}-n_{c}^{2}\right)}{k^{2} k_{4}^{2}+2 i \beta n_{c} k_{x} k_{y, 2}}-\frac{k_{y} k_{2}^{2}\left(k^{2}-n_{c}^{2}\right)}{k^{2}\left(k_{-2}^{2} k_{2}^{2}+2 i \beta n_{c} k_{x} k_{y}\right)}\right] \hat{Q}(\mathbf{k})}{\varepsilon_{c} \int \frac{d^{2} \mathbf{k}}{(2 \pi)^{2}} \frac{n_{c} k_{x}^{2} k_{y, 2}\left(k^{2}-4 n_{c}^{2}\right)}{k^{2}\left(k^{2} k_{4}^{2}+2 i \beta n_{c} k_{x} k_{y, 2}\right)} \hat{Q}(\mathbf{k})-1},
$$

with $k_{y, j} \stackrel{\text { def }}{=} k_{y}+j n_{c} / 2$ and $k_{j}^{2} \stackrel{\text { def }}{=} k_{x}^{2}+k_{y, j}^{2}$ for any integer $j$. At order $\mu^{3}$ the balance is

$$
\mathscr{C}\left(\begin{array}{c}
\bar{u}_{3} \\
C_{3}
\end{array}\right)=\left(\begin{array}{c}
-\partial_{T} \bar{u}_{1} \\
-\partial_{T} C_{1}+\mathscr{N}\left(\bar{u}_{2}, C_{1}\right)+\mathscr{N}\left(\bar{u}_{1}, C_{2}\right)
\end{array}\right) .
$$

If the right-hand side of (B8) is an eigenvector of operator $\mathscr{b}$ with zero eigenvalue, then secular terms appear that produce a mean flow and an associated covariance which diverge at $|y| \rightarrow \infty$. This occurs when

$$
-\partial_{T} \bar{u}_{1}+\mathscr{B}\left\{\mathscr{B}^{-1}\left[-\partial_{T} C_{1}+\mathscr{N}\left(\bar{u}_{2}, C_{1}\right)+\mathscr{N}\left(\bar{u}_{1}, C_{2}\right)\right]\right\}
$$

has a nonzero $e^{i n_{c} y}$ component. The secular terms vanish if

$$
\begin{aligned}
\partial_{T} \bar{u}_{1}+\mathscr{B}\left(\mathscr{B}^{-1} \partial_{T} C_{1}\right)= & f\left(0 \mid \bar{u}_{1}, C^{e}\right)+f\left(0 \mid \bar{u}_{1}, C_{20}\right) \\
& +\mathscr{P}_{1}\left[f \left(0 \mid \bar{u}_{1}, C_{22} e^{2 i n_{c}\left(y_{a}+y_{b}\right) / 2}\right.\right. \\
& \left.+ \text { c.c. })+f\left(0 \mid \bar{u}_{2}, C_{1}\right)\right],
\end{aligned}
$$

where $\mathscr{P}_{1}$ is the operator that projects onto the harmonic $n_{c}$ :

$$
\mathscr{P}_{1} g(y) \stackrel{\text { def }}{=} \int_{\infty} g(s) e^{i n_{c}(y-s)} d s .
$$

Equation (B10) determines the equilibration of the most unstable jet. The terms on the right-hand side of (B10) are nonlinear in $\bar{u}$ and $C$, and they are responsible for the equilibration of the flow-forming SSD instability.
Let us take a closer look into each term of (B10). The second term on the left-hand side of (B10) is

$$
\mathscr{R}\left(\mathscr{B}^{-1} \partial_{T} C_{1}\right)=\left(\partial_{T} A\right)\left(c_{1}-1\right) e^{i n_{c} y},
$$

where

$$
c_{1}=1+\frac{\varepsilon_{c}}{4} \int \frac{d^{2} \mathbf{k}}{(2 \pi)^{2}} \frac{n_{c} k_{x}^{2} k_{y, 1} k_{2}^{2}\left(k^{2}-n_{c}^{2}\right)}{\left(k^{2} k_{2}^{2}+i \beta n_{c} k_{x} k_{y, 1}\right)^{2}} \hat{Q}(\mathbf{k}) .
$$

The first term on the right-hand side of (B10) is the vorticity flux feedback on $\bar{u}_{1}$ at criticality,

$$
f\left(0 \mid \bar{u}_{1}, C^{e}\right)=A e^{i n_{c} y} .
$$

The second term on the right-hand side of (B10) is the vorticity flux feedback between the order $\mu^{1}$ mean jet $\bar{u}_{1}$ and the order $\mu^{2}$ homogeneous eddy covariance $C_{20}$ :

$$
f\left(0 \mid \bar{u}_{1}, C_{20}\right)=-c_{3}^{\mathrm{ec}} A|A|^{2} e^{i n_{c} y},
$$

with

$$
\begin{aligned}
& c_{3}^{\mathrm{ec}} \stackrel{\text { def }}{=} \frac{\varepsilon_{c}}{4} \int \frac{d^{2} \mathbf{k}}{(2 \pi)^{2}} \frac{n_{c} k_{x}^{4} k_{2}^{2}\left(k_{2}^{2}-n_{c}^{2}\right)\left(k^{2}-n_{c}^{2}\right)^{2}}{\left|k^{2} k_{2}^{2}+i \beta n_{c} k_{x} k_{y, 1}\right|^{2}} \\
& \times\left(\frac{2 k_{y, 1}}{k^{2} k_{2}^{2}+i \beta n_{c} k_{x} k_{y, 1}}-\frac{k_{y,-1}}{k^{2} k_{-2}^{2}+i \beta n_{c} k_{x} k_{y,-1}}\right. \\
& \left.-\frac{k_{y, 3}}{k_{2}^{2} k_{4}^{2}+i \beta n_{c} k_{x} k_{y, 3}}\right) \hat{Q}(\mathbf{k}) \text {. }
\end{aligned}
$$


The third term on the right-hand side of (B10) is the $e^{i n_{c} y}$ component of the vorticity flux feedback between the mean jet $\bar{u}_{1}$ with wavenumber $n_{c}$ and the mean jet $\bar{u}_{2}$ with wavenumber $2 n_{c}$ with the inhomogeneous eddy covariance $C_{22}$ and $C_{1}$, respectively:

$$
\begin{aligned}
\mathscr{P}_{1} & {\left[f\left(0 \mid \bar{u}_{1}, C_{22} e^{2 i n_{c}\left(y_{a}+y_{b}\right) / 2}+\text { c.c. }\right)+f\left(0 \mid \bar{u}_{2}, C_{1}\right)\right] } \\
& =-c_{3}^{1,2} A|A|^{2} e^{i n_{c} y},
\end{aligned}
$$

with

$$
\begin{aligned}
c_{3}^{1,2} \stackrel{\text { def }}{=} & \frac{\varepsilon_{c}}{8} \int \frac{d^{2} \mathbf{k}}{(2 \pi)^{2}}\left\{\frac{n_{c} k_{x}^{4} k^{2}\left(k^{2}-n_{c}^{2}\right)\left(k_{2}^{2}-n_{c}^{2}\right)}{\left(k^{2} k_{4}^{2}+2 \mathrm{i} \beta n_{c} k_{x} k_{y, 2}\right)\left(k^{2} k_{2}^{2}+i \beta n_{c} k_{x} k_{y, 1}\right)}\left[\frac{k_{y, 1}\left(k_{4}^{2}-n_{c}^{2}\right)}{k^{2} k_{2}^{2}+i \beta n_{c} k_{x} k_{y, 1}}-\frac{k_{y, 3} k_{4}^{2}\left(k^{2}-n_{c}^{2}\right)}{k^{2}\left(k_{2}^{2} k_{4}^{2}+i \beta n_{c} k_{x} k_{y, 3}\right)}\right] \hat{Q}(\mathbf{k})\right. \\
& \left.+\frac{n_{c} k_{x}^{4} k_{y, 1} k_{2}^{2}\left(k_{-2}^{2}-n_{c}^{2}\right)\left(k^{2}-n_{c}^{2}\right)^{2}\left(k_{-2}^{2} k_{2}^{2}+i \beta n_{c} k_{x} k_{y}\right)}{\left(k^{2} k_{2}^{2}+i \beta n_{c} k_{x} k_{y, 1}\right)^{2}\left(k^{2} k_{-2}^{2}+i \beta n_{c} k_{x} k_{y,-1}\right)\left(k_{-2}^{2} k_{2}^{2}+2 i \beta n_{c} k_{x} k_{y}\right)}\right\} \\
& +i a_{2} \frac{\varepsilon_{c}}{4} \int \frac{d^{2} \mathbf{k}}{(2 \pi)^{2}}\left\{\frac{k^{2}-4 n_{c}^{2}}{k^{2} k_{4}^{2}+2 i \beta n_{c} k_{x} k_{y, 2}}\left[\frac{k_{y, 1}\left(k_{4}^{2}-n_{c}^{2}\right)}{k^{2} k_{2}^{2}+i \beta n_{c} k_{x} k_{y, 1}}-\frac{k_{y, 3} k_{4}^{2}\left(k^{2}-n_{c}^{2}\right)}{k^{2}\left(k_{2}^{2} k_{4}^{2}+i \beta n_{c} k_{x} k_{y, 3}\right)}\right]\right. \\
& \left.+\frac{k^{2}-n_{c}^{2}}{k^{2} k_{2}^{2}-i \beta n_{c} k_{x} k_{y, 1}}\left[\frac{k_{y,-1}\left(k_{2}^{2}-4 n_{c}^{2}\right)}{k^{2} k_{-2}^{2}+i \beta n_{c} k_{x} k_{y,-1}}-\frac{k_{y, 3} k_{2}^{2}\left(k^{2}-4 n_{c}^{2}\right)}{k^{2}\left(k_{2}^{2} k_{4}^{2}+i \beta n_{c} k_{x} k_{y, 3}\right)}\right]\right\} n_{c} k_{x}^{3} \hat{Q}(\mathbf{k}) .
\end{aligned}
$$

Therefore, using (B12), (B14), (B15), and (B17), we can reduce $(\mathrm{B} 10)$ to

$$
c_{1} \partial_{T} A=A-c_{3} A|A|^{2},
$$

where $c_{3} \stackrel{\text { def }}{=} c_{3}^{e c}+c_{3}^{1,2}$.

Finally, we arrive at the G-L equation, (27), by adding the diffusion term $c_{2} \partial_{Y}^{2} A$ on the right-hand side of (B19), with

$$
\begin{aligned}
c_{2} & \stackrel{\text { def }}{=}-\frac{\varepsilon_{c}}{2}\left(\frac{\partial^{2} f}{\partial n^{2}}\right)_{n_{c}, \sigma=0} \\
& =\frac{\varepsilon_{c}}{2} \int \frac{d^{2} \mathbf{k}}{(2 \pi)^{2}}\left[\frac{k_{x}^{2} k_{y, 2}^{2} k^{2}\left(k^{2}-n_{c}^{2}\right)\left(2 k^{2}+\mathrm{i} \beta k_{x}\right)}{\left(k^{2} k_{2}^{2}+i \beta n_{c} k_{x} k_{y, 1}\right)^{3}}-\frac{k_{x}^{2} k^{2}\left(k^{2}-4 n_{c} k_{y}-5 n_{c}^{2}\right)}{2\left(k^{2} k_{2}^{2}+i \beta n_{c} k_{x} k_{y, 1}\right)^{2}}+\frac{n_{c} k_{x}^{2} k_{y, 1}}{k^{2}\left(k^{2} k_{2}^{2}+i \beta n_{c} k_{x} k_{y, 1}\right)}\right] \hat{Q}(\mathbf{k}) .
\end{aligned}
$$

The coefficients $c_{1}, c_{2}$, and $c_{3}$ are all functions of $\beta$, $n_{c}$, and the forcing covariance spectrum $\hat{Q}$. For the ring forcing, (4), considered here, they are all real and positive.

To study the contribution to each of the components of $c_{3}$ from the forced waves with phase lines forming an angle $\vartheta$ with the $y$ axis, we substitute the ring forcing power spectrum, (4). After expressing the integrand in polar coordinates $\left(k_{x}, k_{y}\right)=(k \cos \vartheta, k \sin \vartheta)$ and integrating over $k$, we obtain

$$
\left[c_{3}^{\mathrm{ec}}, c_{3}^{1,2}, c_{3}\right]=\varepsilon_{c} \int_{0}^{\pi / 2}\left[\mathscr{F}_{\mathrm{ec}}, \mathscr{F}_{1,2}, \mathscr{F}_{\mathrm{NL}}\right] d \vartheta
$$

where $\mathscr{F}_{\text {ec }}, \mathscr{F}_{1,2}$, and $\mathscr{F}_{\mathrm{NL}}$ is the contribution of the waves with $\left(k_{x}, k_{y}\right)$ and $\left(-k_{x},-k_{y}\right)$ and their mirror symmetric $\left(-k_{x}, k_{y}\right)$ and $\left(k_{x},-k_{y}\right)$ to the feedbacks, and
$\mathscr{F}_{\mathrm{NL}}=\mathscr{F}_{\text {ec }}+\mathscr{F}_{1,2}$. Figure B1 shows these contributions as a function of wave angle. For $\beta \ll 1$, forced eddies at all angles contribute positively to both $\mathscr{F}_{\text {ec }}$ and $\mathscr{F}_{1,2}$. The eddies tend to reduce the positive destabilizing contribution $\mathscr{F}>0$ at small angles mainly through $\mathscr{F}_{1,2}$, while they enhance the negative stabilizing contribution $\mathscr{F}<0$ at large angles mainly through $\mathscr{F}_{\text {ec }}$. For $\beta \gg 1$, the dominant contribution comes from $\mathscr{F}_{\mathrm{ec}}$, and it follows roughly the same pattern as $\mathscr{F}$. That is, because of the reduction in their energy, the eddies tend to reduce both the upgradient vorticity fluxes of waves with angles $|\vartheta| \lesssim \vartheta_{0}$ and the downgradient fluxes of waves with phase lines at angles $|\vartheta| \gtrsim \vartheta_{0}$, with the latter reduction being larger. As a result, the nonlinear feedback of eddies with phase lines at angles $|\vartheta| \geqq \vartheta_{0}$ is to enhance the jet, and as discussed in section 4 , these are the eddies that support the equilibrated jet. 

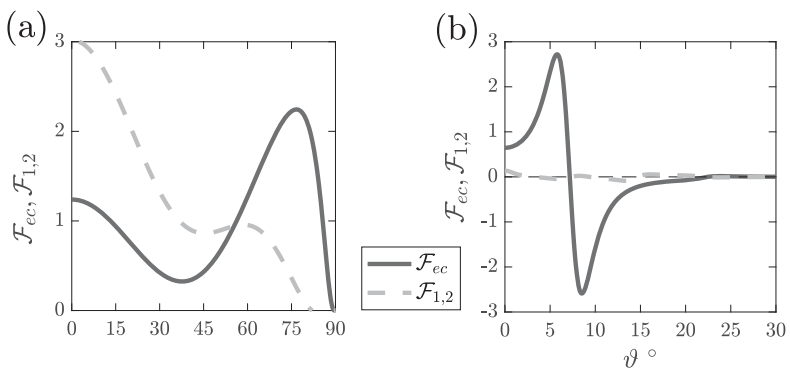

FIG. B1. The contribution of the two feedbacks $\mathscr{F}_{\text {ec }}$ (solid) and $\mathscr{F}_{1,2}$ (dashed) to the nonlinear coefficient $\mathscr{F}_{\mathrm{NL}}$ for the cases with (a) $\beta=0.1$ and (b) $\beta=100$.

\section{APPENDIX C}

\section{Nonisotropic Ring Forcing}

Here we briefly discuss the effect of the forcing anisotropy on the obtained results. Consider the generalization of forcing (4) with spectrum

$$
\hat{Q}_{*}\left(\mathbf{k}_{*}\right)=4 \pi k_{f *} \delta\left(k_{*}-k_{f *}\right)[1+\gamma \cos (2 \vartheta)],
$$

where $\vartheta \stackrel{\text { def }}{=} \arctan \left(k_{y^{*}} / k_{x^{*}}\right)$ and $|\gamma| \leq 1$ so that $\hat{Q}_{*} \geq 0$. Parameter $\gamma$ determines the degree of anisotropy of the forcing. The isotropic case of (4) is recovered for $\gamma=0$. For example, for $\gamma=1$, we get an anisotropic forcing that favors structures with small $\left|k_{y^{*}}\right|$ (i.e., favoring structures like that in Fig. 2a compared to structures like that in Fig. 2b), as if the vorticity injection was due to baroclinic growth processes. All three coefficients $c_{1}, c_{2}$, and $c_{3}$ in (27) are real and positive for forcing $(\mathrm{C} 1)$.

We first note that we obtain similar results to the isotropic-forcing case regarding the comparison of the G-L predictions to the fully nonlinear dynamics (not shown). That is, both the existence of the upper-branch equilibria and the relative quantitative success of the $\mathrm{G}-\mathrm{L}$ dynamics (after the proposed modifications) in predicting the amplitude and instability of the equilibrated jets are insensitive to forcing structure.

Regarding the physical processes underlying the equilibration of the jets, we show in Fig. C1a the amplitude $R_{0}$ for the equilibrated most unstable jet as a function of $\beta$. For $\beta \gg 1$, the amplitude has the same power law as in the isotropic-forcing case shown in Fig. 8a. However, the amplitude shows different dependence with $\beta$ for $\beta \ll 1$; however, this regime is of no interest since, as $\beta \rightarrow 0$, no zonal jets emerge in (1) anyway. The relative contribution of the eddy-correction term and the interaction of $n_{c}$ with the double-harmonic jet in $c_{3}$ is shown in Fig. C1b. Similarly to the isotropic-forcing case, for most values of $\beta$, the equilibration is dominated by the interaction of the most unstable jet with the homogeneous covariance correction.

Last, we note that for anisotropic forcing, a similar qualitative decomposition of $c_{3}$ from various forced waves (as in Fig. 9) also occurs (not shown).

\section{APPENDIX D}

\section{Eckhaus Stability of G-L Dynamics}

To address the Eckhaus instability of the harmonic jet equilibria, we rewrite the jet amplitude $A$ in polar form, (53); we then substitute into (27) and separate real and imaginary parts to obtain

$$
\begin{aligned}
c_{1} \partial_{T} R & =\left[1+c_{2} \partial_{Y}^{2}-c_{2}\left(\partial_{Y} \Theta\right)^{2}\right] R-c_{3} R^{3}, \\
c_{1} R \partial_{T} \Theta & =2 c_{2}\left(\partial_{Y} R\right)\left(\partial_{Y} \Theta\right)+c_{2} R \partial_{Y}^{2} \Theta .
\end{aligned}
$$

Assume now an equilibrium jet with constant amplitude $R_{0}(\nu)$ and a linearly varying phase $\Theta=\nu Y$. Consider small perturbations about this equilibrium jet:

$$
R=R_{0}(\nu)+\rho \quad \text { and } \quad \Theta=\nu Y+\phi,
$$

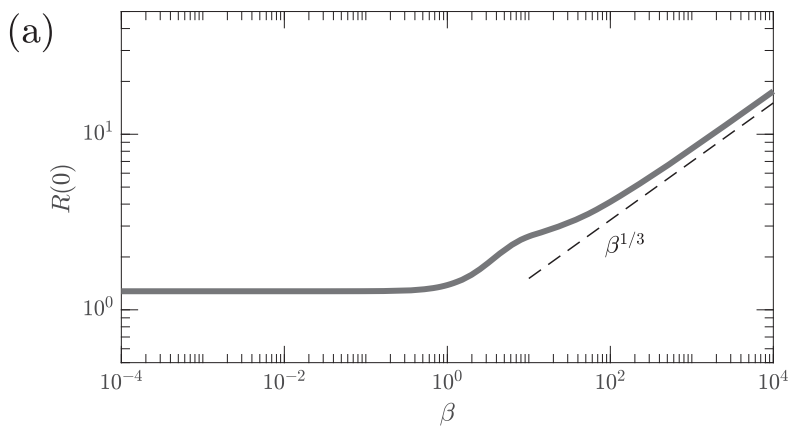

(b)

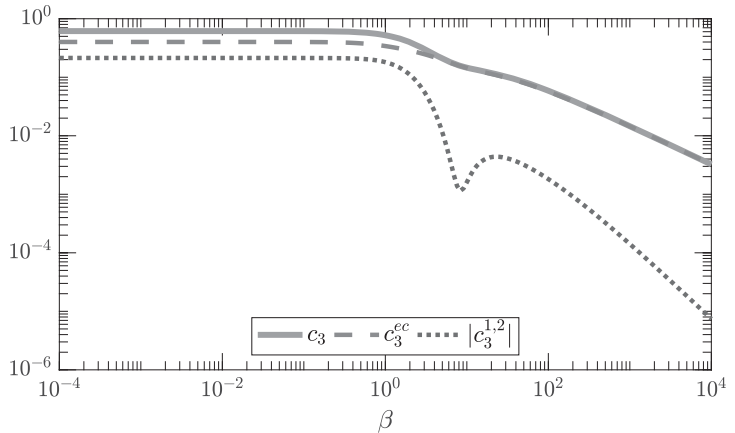

FIG. C1. As in Fig. 8, but for anisotropic forcing (C1) with $\gamma=1$. (a) The amplitude $R_{0}(0)=1 / \sqrt{c_{3}}$ of the equilibrated most unstable jet with wavenumber $n_{c}$ as a function of $\beta$. Dashed line shows the $\beta^{1 / 3}$ slope for reference. (b) The coefficient $c_{3}$ and its decomposition into the contributions $c_{3}^{\text {ec }}$ and $c_{3}^{1,2}$ as a function of $\beta$. 
and linearize (D1) to obtain

$$
c_{1} \partial_{T} \rho=\left[1+c_{2}\left(\partial_{Y}^{2}-\nu^{2}\right)-3 c_{3} R_{0}^{2}\right] \rho-2 c_{2} R_{0} \nu \partial_{Y} \phi
$$

$c_{1} R_{0} \partial_{T} \phi=2 c_{2} \nu \partial_{Y} \rho+c_{2} R_{0} \partial_{Y}^{2} \phi$.

Using the ansatz $[\rho, \phi]=[\hat{\rho}, \hat{\phi}] e^{i q Y+\lambda T}$, we find that the eigenvalues $\lambda$ are

$$
\lambda=\frac{\nu^{2}-\nu_{e}^{2}-q^{2} \pm \sqrt{\left(\nu^{2}-\nu_{e}^{2}\right)^{2}+4 q^{2} \nu^{2}}}{c_{1} \nu_{e}^{2}} .
$$

Instability occurs when $\lambda>0$, that is, when

$$
q^{2}+2\left(\nu_{e}^{2}-3 \nu^{2}\right)<0
$$

\section{REFERENCES}

Ait-Chaalal, F., T. Schneider, B. Meyer, and J. B. Marston, 2016: Cumulant expansions for atmospheric flows. New J. Phys., 18, 025019, https://doi.org/10.1088/1367-2630/18/2/ 025019.

Bakas, N. A., and P. J. Ioannou, 2013a: Emergence of large scale structure in barotropic $\beta$-plane turbulence. Phys. Rev. Lett., 110, 224501, https://doi.org/10.1103/PhysRevLett.110.224501. , and - 2013b: On the mechanism underlying the spontaneous emergence of barotropic zonal jets. J. Atmos. Sci., 70, 2251-2271, https://doi.org/10.1175/JAS-D-12-0102.1.

—, and —, 2014: A theory for the emergence of coherent structures in beta-plane turbulence. J. Fluid Mech., 740, 312341, https://doi.org/10.1017/jfm.2013.663.

$\longrightarrow$, and — 2019a: Emergence of non-zonal coherent structures. Zonal Jets: Phenomenology, Genesis, and Physics, B. Galperin and P. L. Read, Eds., Cambridge University Press, 419-436.

- , and - 2019b: Is spontaneous generation of coherent baroclinic flows possible? J. Fluid Mech., 862, 889-923, https:// doi.org/10.1017/jfm.2018.928.

— N. C. Constantinou, and P. J. Ioannou, 2015: S3T stability of the homogeneous state of barotropic beta-plane turbulence. J. Atmos. Sci., 72, 1689-1712, https://doi.org/10.1175/JAS-D-140213.1.

Bouchet, F., C. Nardini, and T. Tangarife, 2013: Kinetic theory of jet dynamics in the stochastic barotropic and 2D NavierStokes equations. J. Stat. Phys., 153, 572-625, https://doi.org/ 10.1007/s10955-013-0828-3.

__ J. Rolland, and E. Simonnet, 2019: Rare event algorithm links transitions in turbulent flows with activated nucleations. Phys. Rev. Lett., 122, 074502, https://doi.org/10.1103/ PhysRevLett.122.074502.

Chen, S., R. E. Ecke, G. L. Eyink, M. Rivera, M. Wan, and Z. Xiao, 2006: Physical mechanism of the two-dimensional inverse energy cascade. Phys. Rev. Lett., 96, 084502, https://doi.org/ 10.1103/PhysRevLett.96.084502.

Constantinou, N. C., 2015: Formation of large-scale structures by turbulence in rotating planets. Ph.D. thesis, National and
Kapodistrian University of Athens, 193 pp., http://www. didaktorika.gr/eadd/handle/10442/35501?locale $=$ en.

_- and J. B. Parker, 2018: Magnetic suppression of zonal flows on a beta plane. Astrophys. J., 863, 46, https://doi.org/10.3847/ 1538-4357/aace53.

— , B. F. Farrell, and P. J. Ioannou, 2014a: Emergence and equilibration of jets in beta-plane turbulence: Applications of stochastic structural stability theory. J. Atmos. Sci., 71, 18181842, https://doi.org/10.1175/JAS-D-13-076.1.

— A. Lozano-Durán, M.-A. Nikolaidis, B. F. Farrell, P. J. Ioannou, and J. Jiménez, 2014b: Turbulence in the highly restricted dynamics of a closure at second order: Comparison with DNS. J. Phys. Conf. Ser., 506, 012004, https://doi.org/ 10.1088/1742-6596/506/1/012004.

, B. F. Farrell, and P. J. Ioannou, 2016: Statistical state dynamics of jet-wave coexistence in barotropic beta-plane turbulence. J. Atmos. Sci., 73, 2229-2253, https://doi.org/10.1175/ JAS-D-15-0288.1.

Cross, M., and H. Greenside, 2009: Pattern Formation and Dynamics in Nonequilibrium Systems. Cambridge University Press, 552 pp.

Danilov, S., and D. Gurarie, 2004: Scaling, spectra and zonal jets in beta-plane turbulence. Phys. Fluids, 16, 2592-2603, https:// doi.org/10.1063/1.1752928.

Farrell, B. F., and P. J. Ioannou, 2003: Structural stability of turbulent jets. J. Atmos. Sci., 60, 2101-2118, https://doi.org/ 10.1175/1520-0469(2003)060<2101:SSOTJ $>2.0$.CO;2.

- , and -2007 : Structure and spacing of jets in barotropic turbulence. J. Atmos. Sci., 64, 3652-3665, https://doi.org/10.1175/ JAS4016.1.

— the shallow-water equations on an equatorial beta plane. J. Atmos. Sci., 66, 3197-3207, https://doi.org/10.1175/ 2009JAS2941.1.

—_, and ——, 2017: Statistical state dynamics based theory for the formation and equilibration of Saturn's north polar jet. Phys. Rev. Fluids, 2, 073801, https://doi.org/10.1103/ PhysRevFluids.2.073801.

$\longrightarrow,-$ J. Jiménez, N. C. Constantinou, A. Lozano-Durán, and M.-A. Nikolaidis, 2016: A statistical state dynamics-based study of the structure and mechanism of large-scale motions in plane Poiseuille flow. J. Fluid Mech., 809, 290-315, https:// doi.org/10.1017/jfm.2016.661.

,$- \ldots$, and M. A. Nikolaidis, 2017: Instability of the roll-streak structure induced by background turbulence in pretransitional Couette flow. Phys. Rev. Fluids, 2, 034607, https://doi.org/ 10.1103/PhysRevFluids.2.034607.

Fitzgerald, J. G., and B. F. Farrell, 2018a: Statistical state dynamics of vertically sheared horizontal flows in two-dimensional stratified turbulence. J. Fluid Mech., 854, 544-590, https:// doi.org/10.1017/jfm.2018.560.

— forming instability in stratified turbulence: Analytical linear stability analysis of statistical state dynamics equilibria. J. Atmos. Sci., 75, 4201-4227, https://doi.org/10.1175/JASD-18-0075.1.

— , and - 2019: Statistical state dynamics analysis of buoyancy layer formation via the Phillips mechanism in twodimensional stratified turbulence. J. Fluid Mech., 864, R3, https://doi.org/10.1017/jfm.2019.72.

Frishman, A., and C. Herbert, 2018: Turbulence statistics in a 2D vortex condensate. Phys. Rev. Lett., 120, 204505, https:// doi.org/10.1103/PhysRevLett.120.204505. 
_ J. Laurie, and G. Falkovich, 2017: Jets or vortices-What flows are generated by an inverse turbulent cascade? Phys. Rev. Fluids, 2, 032602, https://doi.org/10.1103/PhysRevFluids.2.032602.

Holloway, G., 2010: Eddy stress and shear in 2-D flows. J. Turbul., 11, N14, https://doi.org/10.1080/14685248.2010.481673.

Hoyle, R., 2006: Pattern Formation: An Introduction to Methods. Cambridge University Press, 422 pp.

Huang, H.-P., and W. A. Robinson, 1998: Two-dimensional turbulence and persistent zonal jets in a global barotropic model. J. Atmos. Sci., 55, 611-632, https://doi.org/10.1175/ 1520-0469(1998)055<0611:TDTAPZ > 2.0.CO;2.

Ingersoll, A. P., 1990: Atmospheric dynamics of the outer planets. Science, 248, 308-315, https://doi.org/10.1126/ science.248.4953.308.

_ - and Coauthors, 2004: Dynamics of Jupiter's atmosphere. Jupiter: The Planet, Satellites, and Magnetosphere, F. Bagenal, T. E. Dowling, and W. B. McKinnon, Eds., Cambridge University Press, 105-128.

Kraichnan, R. H., 1976: Eddy viscosity in two and three dimensions. J. Atmos. Sci., 33, 1521-1536, https://doi.org/10.1175/ 1520-0469(1976)033<1521:EVITAT>2.0.CO;2.

Manfroi, A. J., and W. R. Young, 1999: Slow evolution of zonal jets on the beta plane. J. Atmos. Sci., 56, 784-800, https:// doi.org/10.1175/1520-0469(1999)056<0784:SEOZJO > 2.0.CO;2.

Marston, J. B., E. Conover, and T. Schneider, 2008: Statistics of an unstable barotropic jet from a cumulant expansion. J. Atmos. Sci., 65, 1955-1966, https://doi.org/10.1175/ 2007JAS2510.1.

— G. P. Chini, and S. M. Tobias, 2016: Generalized quasilinear approximation: Application to zonal jets. Phys. Rev. Lett., 116, 214501, https://doi.org/10.1103/PhysRevLett.116.214501.

_ - W. Qi, and S. M. Tobias, 2019: Direct statistical simulation of a jet. Zonal Jets: Phenomenology, Genesis, and Physics, B. Galperin and P. L. Read, Eds., Cambridge University Press, 332-346.

Parker, J. B., and J. A. Krommes, 2013: Zonal flow as pattern formation. Phys. Plasmas, 20, 100703, https://doi.org/10.1063/ 1.4828717.
— and - 2014: Generation of zonal flows through symmetry breaking of statistical homogeneity. New J. Phys., 16, 035006, https://doi.org/10.1088/1367-2630/16/3/035006.

Read, P. L., Y. H. Yamazaki, S. R. Lewis, P. D. Williams, R. Wordsworth, K. Miki-Yamazaki, J. Sommeria, and H. Didelle, 2007: Dynamics of convectively driven banded jets in the laboratory. J. Atmos. Sci., 64, 4031-4052, https://doi.org/ 10.1175/2007JAS2219.1.

Rhines, P. B., 1975: Waves and turbulence on a beta-plane. J. Fluid Mech., 69, 417-433, https://doi.org/10.1017/S0022112075001504.

Salyk, C., A. P. Ingersoll, J. Lorre, A. Vasavada, and A. D. Del Genio, 2006: Interaction between eddies and mean flow in Jupiter's atmosphere: Analysis of Cassini imaging data. Icarus, 185, 430-442, https://doi.org/10.1016/j.icarus.2006. 08.007.

Shepherd, T. G., 1987: Rossby waves and two-dimensional turbulence in a large-scale zonal jet. J. Fluid Mech., 183, 467-509, https://doi.org/10.1017/S0022112087002738.

Srinivasan, K., and W. R. Young, 2012: Zonostrophic instability. J. Atmos. Sci., 69, 1633-1656, https://doi.org/10.1175/JAS-D-11-0200.1.

Starr, V. P., 1968: Physics of Negative Viscosity Phenomena. McGraw Hill, 256 pp.

Thomas, V., B. K. Lieu, M. R. Jovanović, B. F. Farrell, P. J. Ioannou, and D. F. Gayme, 2014: Self-sustaining turbulence in a restricted nonlinear model of plane Couette flow. Phys. Fluids, 26, 105112, https://doi.org/10.1063/1.4898159.

Tobias, S. M., and J. B. Marston, 2013: Direct statistical simulation of out-of-equilibrium jets. Phys. Rev. Lett., 110, 104502, https://doi.org/10.1103/PhysRevLett.110.104502.

, K. Dagon, and J. B. Marston, 2011: Astrophysical fluid dynamics via direct numerical simulation. Astrophys. J., 727, 127, https://doi.org/10.1088/0004-637X/727/2/127.

Vallis, G. K., and M. E. Maltrud, 1993: Generation of mean flows and jets on a beta-plane and over topography. J. Phys. Oceanogr., 23, 1346-1362, https://doi.org/10.1175/1520-0485(1993) $023<1346$ :GOMFAJ $>2.0 . \mathrm{CO} ; 2$.

Vasavada, A. R., and A. P. Showman, 2005: Jovian atmospheric dynamics: An update after Galileo and Cassini. Rep. Prog. Phys., 68, 1935-1996, https://doi.org/10.1088/0034-4885/68/8/R06. 